\title{
Cognitive Deficits and Altered Cholinergic Innervation in Young Adult Mice Carrying a Parkinson's Disease LRRK2-G2019S Knockin Mutation
}

\author{
by \\ Ayan Hussein, Alexander Tielemans, Mark G. Baxter, Deanna L. Benson and George \\ W. Huntley* \\ Nash Family Department of Neuroscience, Friedman Brain Institute and the Graduate \\ School of Biomedical Sciences, Icahn School of Medicine at Mount Sinai, New York, NY \\ 10029
}

\begin{abstract}
*To whom correspondence should be addressed: george.huntley@mssm.edu or deanna.benson@mssm.edu
\end{abstract}

Running title: Cognitive and cholinergic deficits in LRRK2-G2019S mice

Keywords: 5-CSRT task, attention, acetylcholine, cognitive flexibility, goal-directed learning, non-motor symptoms

Pages: $40 \quad$ Figs: $9 \quad$ Tables: 0

Conflict of Interest: The authors declare no competing financial conflicts.

Acknowledgements: This work was supported by the National Institutes of Health, NINDS grant numbers F31NS117089 and R01NS107512. We thank Lydia Gould and Pamela Del Valle for expert technical assistance. Microscopy was performed at the Microscopy Core and Advanced Bioimaging Center at the Icahn School of Medicine at Mount Sinai. 


\begin{abstract}
Impaired executive function is a common and debilitating non-motor symptom of idiopathic and hereditary Parkinson's disease (PD), but there is little understanding of the underlying pathophysiological mechanisms and circuits. The G2019S mutation in the kinase domain of leucine-rich repeat kinase 2 ( $L R R K 2)$ greatly increases risk for late-onset PD, and non-manifesting LRRK2-G2019S carriers also exhibit early and significant cognitive impairment. Here, we subjected young adult mice carrying a Lrrk2G2019S knockin mutation to touchscreen-based operant tasks that measure attention, goal-directed learning and cognitive flexibility, all of which rely on prefrontal-striatal connectivity and are strongly modulated by cholinergic innervation. In a visuospatial attention task, mutant mice exhibited significantly more omissions and longer response latencies than controls that could not be attributed to deficits in motivation, visual sensory perception per se or locomotion, thereby suggesting impairment in divided attention and slower information processing speed. Pretreating mice with the acetylcholinesterase inhibitor donepezil normalized both higher omission rates and longer reward latencies in the mutants, but did not affect any performance metric in controls. Strikingly, cholinergic fiber density in MPFC and dorsomedial striatum was significantly sparser in mutants than in controls, while further behavioral interrogation of the mutants revealed significant impairments in action-outcome associations but preserved cognitive flexibility. These data suggest that the G2019S mutation impacts cholinergic innervation and impairs corticostriatal network function in young adulthood that may contribute to early PD-associated cognitive deficits.
\end{abstract}




\section{STATEMENT OF SIGNIFICANCE}

The LRRK2-G2019S mutation causes hereditary Parkinson's disease and is found in some idiopathic cases. Early cognitive impairment is a common symptom of hereditary and idiopathic PD, yet there is little mechanistic understanding of such impairment. Here, we tested young adult Lrrk2-G2019S knockin mice in a series of touchscreen-based visuospatial tasks. We found that mutants exhibited significant deficits in attention and goal-directed learning, and had significantly slower information processing speed. Treatment with an acetylcholinesterase inhibitor reversed some of these behavioral deficits, while anatomical analyses showed significantly sparser cholinergic innervation of brain structures important for executive function. These findings suggest the G2019S mutation alters cholinergic signaling in young adulthood, and thus may contribute to early PD-associated impairment in several cognitive domains. 


\section{INTRODUCTION}

Cognitive impairment affects $30-50 \%$ of those with Parkinson's disease (PD), with a greater prevalence in males and an onset that can precede motor symptoms (Muslimovic et al., 2005; Cammisuli et al., 2019). Deficits include impaired attention, cognitive flexibility, perceptual decision making, reinforcement learning and generally slower information processing speed, and can progressively worsen over time leading to dementia (Robbins and Cools, 2014; Perugini et al., 2018). The negative impact of PD-associated cognitive impairment on quality of life is significant, often exceeding that resulting from motor disturbances (Prakash et al., 2016). Nevertheless, there is little understanding of pathophysiological mechanisms and circuits that underlie PDassociated cognitive impairment, hindering treatment options.

In primates and rodents, executive function requires prefrontal cortex and connectivity with striatum (Selemon and Goldman-Rakic, 1988; Yin et al., 2005; Ragozzino, 2007). Humans with early PD-associated impairment in executive function show altered frontal-striatal network activity (Lewis et al., 2003; Wolters et al., 2019). Additionally, executive function, particularly attention, is strongly modulated by cholinergic innervation of prefrontal cortex and striatum (Everitt and Robbins, 1997; Baxter and Chiba, 1999; Ballinger et al., 2016). Cholinergic innervation of prefrontal cortex arises from neurons in basal forebrain and from cortical cholinergic interneurons (Ballinger et al., 2016; Obermayer et al., 2019), while cholinergic innervation of striatum arises largely from striatal cholinergic interneurons with a sparse contribution from neurons in mesopontine tegmental nuclei (Bolam et al., 1984; Dautan et al., 2016). While much focus of PD research is dopamine neuron degeneration, cholinergic neurons also degenerate in humans during the course of PD (Choi et al., 2012; Pasquini et al., 2021). Progressive loss of cortical cholinergic projections is associated with cognitive impairment in non-demented PD patients (Bohnen et al., 2015; Kim et al., 2017) and has been associated with PD-related deficits in attention and executive function (Bohnen et al., 2006). Thus, while dysfunctional cholinergic signaling is implicated in PD-related cognitive decline, there is little understanding of how, when or where changes in cholinergic signaling arise. 
One approach to address such lapses is to experimentally interrogate animals carrying genetic mutations that in humans cause PD (Dawson et al., 2010). The G2019S autosomal dominant mutation in leucine-rich repeat kinase 2 (LRRK2) is the most frequent cause of familial PD and is also found in some idiopathic PD cases (Martin et al., 2014). LRRK2 is a large protein with a catalytic core comprising GTPase and kinase domains. The G2019S mutation, located in the kinase domain, increases kinase activity (Paisan-Ruiz et al., 2013). Human G2019S carriers develop late-onset PD with a clinical presentation similar to idiopathic PD, suggesting common pathophysiological mechanisms (Martin et al., 2014). Consistent with this, G2019S carriers also exhibit early cognitive impairment, although this tends to be less severe than idiopathic PD (Healy et al., 2008; Thaler et al., 2012; Gaig et al., 2014; Mirelman et al., 2015; Ben Romdhan et al., 2018; Piredda et al., 2020) and, prior to the onset of motor symptoms, exhibit altered functional coupling between cortex and striatum (Thaler et al., 2013; Helmich et al., 2015; Bregman et al., 2017). Additionally, human G2019S carriers with or without PD exhibit altered acetylcholinesterase activity in cortical and subcortical regions in comparison with idiopathic PD or healthy controls (Liu et al., 2018).

In human and rodent brains, LRRK2 expression is highest in striatum and cortex, with low expression in substantia nigra pars compacta or basal forebrain (Benson and Huntley, 2019). Developmental studies of postnatal mice show that LRRK2 expression levels in cortex and striatum rise contemporaneously with corticostriatal synaptogenesis (Giesert et al., 2013), suggesting that the mutation may shape development of circuits critical for executive function in ways that negatively impact cognitive abilities later in life (Hussein et al., 2021). Accordingly, in this study, we use G2019S knockin mice to test the prediction that executive function across several cognitive domains is aberrant by young adulthood, and explore functional and anatomical aberrations in cholinergic innervation as a contributing factor, both of which have not previously been rigorously explored in PD mouse models. 


\section{MATERIALS AND METHODS}

Mice. PD is more common in males than in females and at the time these studies were initiated, most evidence supported that early cognitive decline in idiopathic PD may be worse in males than in females (Elbaz et al., 2016; Reekes et al., 2020). Accordingly, the present study used male Lrrk2-G2019S knockin (GSKI) mice (RRID:MGI:6273311) that were congenic on a C57BI/6NTac background. It is worth noting, however, that some newer work suggests that within PD patients, cognitive impairment in male LRRK2-G2019S carriers may be less severe than in female carriers (Ben Romdhan et al., 2018). In any event, over the course of all experiments, mice were aged 2-6 postnatal months. Age- and strain-matched wildtype (WT) mice served as controls. All mice were bred and raised under identical conditions in the vivarium. The GSKI mice were generated by Eli Lily and characterized previously (Matikainen-Ankney et al., 2016, 2018; Guevara et al., 2020). They exhibit normal life spans and body weights, express normal levels of tyrosine hydroxylase in the striatum, and express normal physiological levels of LRRK2-G2019S protein in brain. GSKI mice were backcrossed to commercial WT C57BI/6NTac mice every fourth generation to prevent genetic drift. Genotypes were confirmed by PCR and sequencing. In humans, the G2019S mutation is autosomal dominant, producing similar disease onset and progression in heterozygous and homozygous mutation carriers (Zimprich et al., 2004; Paisán-Ruiz et al., 2013). As predicted from this, previous studies showed similar behavioral and electrophysiological outcomes in heterozygous and homozygous G2019S mice (Matikainen-Ankney et al., 2016, 2018; Guevara et al., 2020). We thus used heterozygous G2019S mutants for these experiments. All procedures were approved by Mount Sinai's Institutional Animal Care and Use Committee and conformed to guidelines established by the National Institutes of Health.

Behavioral tasks. Mice were 2- to 3-months old when behavioral experiments began. They were housed in groups of 3-4 in a temperature-controlled room with a $12 \mathrm{hr}$ light/dark cycle (lights on at 7:00 am). Behavioral testing was conducted once a day for $5-6$ days a week during the light phase of the cycle. Mice were food restricted to $85 \%$ of their free-feeding weight. Water was available ad libitum, and food rations, adjusted 
based on body weight, were given at the end of each day.

Apparatus. All behavioral tasks were carried out using four automated Bussey-Saksida style touchscreen-based operant chambers (Lafayette Instruments, Lafayette, IL) consisting of four modular testing chambers housed individually within a sound- and light-attenuating cubicle equipped with an overhead camera to monitor task-related locomotion. Inside each chamber, a reward collection magazine connected to a liquid dispenser pump was situated opposite a touch-sensitive monitor. Depending on the task, the touchscreen monitor was divided into one, two or five response windows by interchanging different black Perspex masks. Each response window was 4.0 x $4.0 \mathrm{~cm}$ regardless of how many response windows each mask contained. The collection magazine contained an LED illuminated coincident with delivery of a liquid reward (Nesquik® strawberry-flavored milk) and a tone. Infrared beam arrays distributed in front of the touchscreen, reward collection port and across the intervening floor, were used to monitor locomotor. Different training schedules, depending on the task, were designed and data were analyzed using ABET II Touch software (Lafayette Instruments), while inputs and outputs from all four chambers were controlled by WhiskerServer software (Lafayette Instruments).

5-CSRT task. This task was conducted on 18 GSKI mice and 19 WT mice and followed established procedures (Bari et al., 2008; Mar et al., 2013). The following performance metrics were collected (and defined as): \% response accuracy (\# correct responses/(total number of correct and incorrect responses) x 100); \% correct responses (\# correct responses/(total number of correct, incorrect and omitted responses) x 100); \% omissions (\# of omissions/(total number of correct, incorrect and omitted responses) $\mathrm{x}$ 100); \# trials (\# correct and incorrect trials); correct response latency (the elapsed time between stimulus offset following a correct touch and head entry into the reward magazine signaled by a back IR beam break).

Pre-training. Mice progressed through 5 pretraining stages (habituation, initial touch, must touch, must initiate and punish incorrect) before advancing to training. In stage 1 , they were acclimated to the chambers for a 40 min session with 
the tray-light turned on, and the tray was primed with strawberry milk. In stages 2 and 3, a mask with five response windows was placed over the touchscreen, and mice learned to nose poke the stimulus (white square) displayed randomly in one of the five response windows to elicit strawberry milk delivery. In stage 4, mice learned to initiate each trial via head entry into the magazine tray; withdrawal from the magazine elicited the next stimulus presentation. Mice were required to complete 30 trials in 60 minutes across two consecutive days to meet completion criteria for stages 2-4. In stage 5, mice were discouraged from nose-poking blank locations on the screen by imposing a 5 sec timeout period signaled by illumination of the house light. Mice were rewarded with $7 \mu \mathrm{l}$ of strawberry milk at all stages. To progress to task training, mice were required to complete 24 out of 30 trials in 60 minutes (80\% accuracy) across two out of three consecutive days.

Training. Daily training sessions lasted $60 \mathrm{~min}$. At the beginning of each session, the magazine light was illuminated and a free strawberry milk reward was dispensed. A nose poke entry into the reward port extinguished the magazine light, and upon exiting initiated a $5 \mathrm{sec}$ intertrial interval (ITI). Following the ITI, a visual stimulus (white square) was randomly displayed in 1 of 5 locations on the screen. A nose poke response made in the corresponding location either during the stimulus presentation or the following $5 \mathrm{sec}$ limited hold period (stimulus offset) was deemed correct and resulted in strawberry milk delivery. The stimulus duration was initially set at 32 seconds and gradually reduced to 16, 8, 4, and 2 sec to establish baseline performance at $2 \mathrm{sec}$ stimulus duration. The performance criteria were completion of 50 out of 60 trials within $60 \mathrm{~min}$, response accuracy $\geq 80 \%$, and trial omissions $\leq 20 \%$, across three of four consecutive days.

Probe trials. Once all mice were performing at baseline (2 sec stimulus duration), they were subjected to two probe trials in which attentional demand was increased by shortening stimulus durations. In probe trial 1 ( $n=19$ WT mice, 17 GSKI mice), stimulus durations were (in seconds): $1.5,1.0,0.8,0.6$, and 0.4 , where mice were tested at a single stimulus duration for two consecutive days (within session). The order of stimulus durations presented to each mouse was randomized in a Latin square design. In between each of the shortened stimulus durations, mice were tested 
at the baseline $2 \mathrm{sec}$ stimulus duration until performance criteria were reattained (completion of 50 out of 60 trials, $\geq 80 \%$ accuracy and $\leq 20 \%$ omissions in $60 \mathrm{~min}$ ). In probe trial 2 ( $\mathrm{n}=13$ WT mice, $11 \mathrm{GSKI}$ mice), stimulus durations were (in seconds): 2.0, 1.0, 0.8 and 0.6 in a within-session block design for two consecutive days. Equal numbers of each of the four stimulus durations were randomly presented during the 60-trial session over a 60 min period.

Visual Perception test. We tested visual perceptual abilities of mice at the shortest stimulus duration used in the 5-CSRT task $(0.4 \mathrm{sec})$ in a subset of mice completing the 5-CSRT task ( $\mathrm{n}=13$ WT mice; 9 GSKI mice), under conditions where attentional demand was lowered by presenting the stimulus in one response window, thereby eliminating the need for mice to divide their attention across 5 response windows. In this 1-choice version of the task, the stimulus (white square) was only presented in the middle window of the five-response window mask. Other task parameters and the primary response rules remained the same. Once mice reattained the baseline performance at $2 \mathrm{sec}$, they were tested on the $0.4 \mathrm{sec}$ stimulus duration (50 out of 60 trials within $60 \mathrm{~min}$ ) for two consecutive days.

Administration of donepezil. These experiments were conducted on a subset of WT $(n=13)$ and GSKI (n=9) mice after completing the two probe trials. Mice received either donepezil or vehicle (normal saline) using a within-subject design where, for each mouse, the order of treatment with vehicle or donepezil was counterbalanced across animals of each group. Donepezil hydrochloride $(0.3 \mathrm{mg} / \mathrm{kg}$; Bio-Techne Corp, Minneapolis, MN) was dissolved in $0.9 \%$ saline and was made fresh each day. The donepezil dose used was based on prior studies (Yuede et al., 2007; Bartko et al., 2011; Romberg et al., 2011). Mice were injected intraperitoneally one hour before behavioral testing on each day of a two-day testing sequence in the 5-CSRT task. Testing consisted of 50 trials for 2 consecutive days at a stimulus duration of $0.4 \mathrm{sec}$. In between dosing/testing sequences, mice received three drug-free days over which they were tested using a baseline $2 \mathrm{sec}$ stimulus duration; animals were required to reinstate baseline performance ( $\geq 80 \%$ accuracy, $<20 \%$ omissions).

Instrumental conditioning and outcome devaluation. Action-outcome learning was assessed in a second, separate cohort of mice ( $n=7$ WT and 6 GSKI mice) by adapting 
for touchscreens a previously described 4-day lever-pressing paradigm of instrumental conditioning followed by subsequent tests of reward devaluation to discriminate goaldirected and habitual responding (Shan et al., 2014). In this task, a one responsewindow mask was used. Training consisted of two 60-minute sessions of habituation in the chamber as described above, followed by a $2 \mathrm{hr}$ session using a continuous reinforcement (CRF) schedule, where each nose poke to the stimulus window elicited a reward. Following training, mice progressed to a 4-day testing protocol, where each session was limited to $60 \mathrm{~min}$. On day 1, mice were tested on a CRF schedule. On days 2-4, mice were subjected to a random interval (RI) session of 15, 30, and $30 \mathrm{sec}$. On days 5 and 6 , outcome devaluation tests were conducted. On day 5 , half of the mice were given $1 \mathrm{hr}$ free access to strawberry milk reward (devalued condition), while the other half got $1 \mathrm{hr}$ free access to home-cage chow (non-devalued condition), followed immediately by a 5 min extinction test. No reward was elicited in this extinction test. On day 6 , a second extinction test was performed in the same manner as the firstexcept that the satiety conditions were reversed.

Pairwise visual discrimination and reversal learning. Cognitive flexibility was tested in a third, separate cohort ( $\mathrm{n}=5$ WT and 7 GSKI mice) using pairwise visual discrimination and reversal learning. Before testing, mice underwent touchscreen training as described above, but using a mask containing two response windows. In the initial discrimination phase, mice were presented simultaneously with two distinct visual stimuli (plane vs. spider) and taught to associate one stimulus (e.g. plane) with strawberry milk delivery and the other (e.g. spider) with a $5 \mathrm{sec}$ timeout period signaled by illumination of the house light (Bartko et al., 2011). The rewarded stimulus was pseudo-randomly displayed in one of the two response windows and was counterbalanced within mice. An incorrect choice resulted in a correction trial in which the stimulus display was repeated in the same window until the mouse made a correct response. However, correction trials were not included in the total tally of correct trials. Upon completing 24 out of 30 trials ( $80 \%$ accuracy) within 60 mins in two consecutive days, mice advanced to the subsequent reversal phase in which reward contingencies were switched. As with the discrimination phase, an incorrect choice resulted in a 
correction trial in which the stimulus display was repeated in the same window until the mouse made a correct response. Measurements included the rate at which mice acquired the new stimulus-reward associations as well as the number of correction responses in each phase (Bussey et al., 1997).

Progressive ratio task. Motivation for the reward was evaluated by a progressive ratio test of subsets of mice following completion of the 5-CSRT task ( $n=13$ WT and 11 GSKI mice) or instrumental conditioning/outcome devaluation ( $n=4$ WT and 4 GSKI mice). Mice were subjected to fixed ratio (FR) schedules in which nose-poking the illuminated center response window for a fixed number of times (FR 1, 2, 3, or 5) was required to elicit a strawberry milk reward. Criterion to advance was completion of 30 trials within 60 min for two consecutive days. Following completion of FR5, mice advanced to a progressive ratio (PR) 4 testing schedule, where reward response requirement incremented on a linear +4 basis (i.e., 1, 5, 9, 13, etc.) in each subsequent trial. Sessions were run for 60 minutes and were terminated when a mouse ceased responding for 5 minutes (the breakpoint).

Statistical analysis of behavioral data. Statistical analyses were performed in $\mathrm{R}$ version 4.1.2 (R Core Team, 2021). Statistical tests for each dataset are specified within each figure legend. For most analyses we used a linear mixed models regression framework with genotype and behavioral variables (e.g. stimulus duration) or treatment levels (donepezil/saline) as fixed effects and mouse as a random effect, using $\mathrm{R}$ packages Ime4 (Bates et al., 2015) and ImerTest (Kuznetsova et al., 2017) and the mean score in each session as the unit of analysis. These models were evaluated with F-tests on type III sums of squares on fixed effects and their interactions. Where significant effects were indicated, these were decomposed through comparisons of estimated marginal means using the emmeans package (Lenth, 2022). Data are reported as mean \pm standard error of the mean (SEM) and all significant comparisons are displayed by asterisks in the figures. 
Immunolabeling. Behaviorally naïve male mice ( $\mathrm{n}=3 \mathrm{WT}, 3 \mathrm{GSKI}$ mice, aged 2-3 months) were anesthetized with a mixture of ketamine $(90 \mathrm{mg} / \mathrm{kg})$ and xylazine $(20$ $\mathrm{mg} / \mathrm{kg}, \mathrm{IP}$ ) and transcardially perfused first with cold $1 \%$ paraformaldehyde (PFA) in 0.1M phosphate buffered saline (PBS, pH 7.3) for $30-45 \mathrm{sec}$ followed by cold $4 \%$ PFA for 10 min. Brains were dissected, post-fixed for 6-8 hours in 4\% PFA, then soaked in $7 \%$ sucrose in PBS for 24 hrs. Serial coronal tissue sections through mPFC (including areas $\mathrm{PL} / \mathrm{IL}$ ) and dorsal striatum were cut on a vibratome at a setting of $50 \mu \mathrm{m}$. Tissue sections were then permeabilized with $0.25 \%$ Triton X-100 in PBS for 15 min, washed thoroughly in PBS, and incubated in a PBS solution containing $10 \%$ Bovine Serum Albumin (BSA) for $1.5 \mathrm{~h}$ at room temperature (RT). Sections were then incubated for 72 hrs at $4^{\circ} \mathrm{C}$ in primary rabbit anti-vesicular acetylcholine transporter (VAChT) antisera (Synaptic Systems; 1:500, RRID_AB887864) containing 2\% BSA and 0.1\% Triton X100 in PBS. Sections were washed thoroughly in PBS, followed by RT incubation for 1 hr with secondary donkey anti-rabbit Alexa 488 (Jackson ImmunoResearch; 1:500, RRID_AB2313584). After washing, sections were mounted using VectaShield antifade mounting media and counterstained with DAPI. Coverslip edges were sealed with nail polish and dried overnight in the dark.

Image analysis. Analysis was carried out on three immunolabeled sections through areas PL/IL and three immunolabeled sections through dorsomedial striatum from each mouse. No attempt was made to analyze PL and IL separately. A series of contiguous single optical plane images through PL/IL or striatum were acquired with a $40 \mathrm{X}$ objective on a Zeiss LSM 780 confocal microscope and stitched together using Zeiss Zen SP1 (black) software (v.8.1.11.484) to create a single tiled image per section through each structure (tiled image dimensions in PL/IL: $1020 \mu \mathrm{m} \times 1226 \mu \mathrm{m}$, spanning layers 1-6; tiled image dimension in DMS: $820 \mu \mathrm{m} \times 820 \mu \mathrm{m}$ ). Image acquisition parameters were kept identical for all sections from mice of each genotype. A sampling frame $\left(207.5 \mu \mathrm{m}^{2}\right)$ was used to define a region of interest (ROI) and overlayed onto the raw tiled images of PL/IL (6 ROIs pers section, sampling superficial-to-deep cortical layers) and dorsomedial striatum (3 ROls per section). VAchT fiber density, average size of immunolabeled puncta and immunofluorescent intensity were determined using 
ImageJ by applying a thresholding function to each ROI to create a binarized mask of the immunofluorescent signal. The mask was then applied to the original image to capture and quantify density, area and intensity of suprathreshold immunofluorescent pixels. The density of VAChT immunolabeled fibers was expressed as the percent of ROI area occupied by suprathreshold pixels (Dougherty et al., 2020). Statistical comparisons between groups were made using a linear mixed model on each measure, with region and genotype as fixed effects and mouse as a random effect; $p$ values < 0.05 were considered statistically significant.

\section{RESULTS}

Cohorts of young adult male wildtype (WT) and Lrrk2-G2019S knockin (GSKI) mice were subjected to visuospatial tasks that evaluate attention, goal-directed learning and cognitive flexibility using automated, standardized touch screen-based operant chambers.

\section{The 5-CSRT task reveals an attention deficit in GSKI mice}

Task acquisition consisted of an initial training phase in which mice learned to respond to a stimulus presented on a screen for a limited time. In the training phase, stimulus duration was initially $32 \mathrm{sec}$, then sequentially reduced to a baseline of $2 \mathrm{sec}$. Performance criteria for advancing to the next shorter stimulus duration were completion of $\geq 50$ trials within a 60 min session, with response accuracy of $\geq 80 \%$ and an omission rate of $\leq 20 \%$ across 3 out of 4 consecutive sessions (Mar et al., 2013). All WT and GSKI mice reached task criteria at each stimulus duration. However, while GSKI mice displayed similar percentages of correct responses at each stimulus duration (Fig. 1A), they made more omissions (Fig. 1B) and completed fewer trials (Fig. 1D) compared to WT mice. GSKI mice exhibited longer latencies compared to WT mice at the longer stimulus durations during initial training (Fig. 1E). During the ITI, GSKI mice made fewer premature responses compared to WT mice (Fig. 1F). Additionally, GSKI mice exhibited greater response accuracy (correct trials / correct + incorrect trials) in comparison with WT mice (Fig. 1C), perhaps reflecting a tendency to fail to respond (make an omission) versus making an incorrect response on trials where there was a 
lapse in attention. There were no significant differences between genotypes in perseverative responses following a correct choice at any stimulus duration (genotype, $F_{1,35}=0.64, p=0.43$, stimulus duration $x$ genotype interaction: $F_{4,510}=0.72, p=0.58$ ).

Once all mice achieved stable performance at baseline (2 sec stimulus duration), cognitive abilities were challenged in two probe trials. Poorer performance by the GSKI mice at the shortest stimulus durations also suggested attentional deficits, even after they had reached criterion on the basic task. In probe 1 (between-session design), entire sessions with stimulus durations of 1.5, 1.0, 0.8, 0.6 and $0.4 \mathrm{sec}$ were presented in a random session order to prevent order effects. Mice were tested on a single stimulus duration for two consecutive days, interleaved with testing for two consecutive days at baseline (2 sec stimulus duration). In probe 1, GSKI mice showed a significantly greater percentage of omissions (Fig. 2A), a significantly lower percentage of correct responses (Fig. 2B) and completed significantly fewer trials (Fig. 2D) compared to WT mice. GSKI mice showed slightly worse response accuracy at the shortest stimulus duration (Fig. 2C). Additionally, GSKI mice showed a longer correct response latency at $0.6 \mathrm{sec}$ and $0.4 \mathrm{sec}$ (Fig. 2E). Moreover, there was a main effect of genotype on premature responding, with GSKI mice making significantly fewer premature responses than WT mice (Fig. 2F). There were no significant genotype differences in perseverative responses at any stimulus duration (genotype, $F_{1, \sim 37.4}=1.06, p=0.31$, stimulus duration $x$ genotype interaction: $F_{5, \sim 676.4}=0.5, p=0.77$ ).

In probe 2 (within-session design), stimulus durations of 2.0, 1.0, 0.8, and $0.6 \mathrm{sec}$ were presented in random order within a session, with each stimulus duration presented an equal number of times per session. Similar to probe 1, GSKI mice displayed a significant increase in percent omissions (Fig. 3A) and a decrease in percent correct responses (Fig. 3B) at the shortest stimulus duration in comparison with WT mice. There was no significant difference in percent response accuracy (Fig. 3C), average correct response latencies (Fig. 3D), premature responses (Fig. 3E), or perseverative errors following correct responses (Fig. 3F) for the GSKI mice compared to WT mice.

Deficits in 5-CSRT task not attributable to diminished motivation, locomotion or impaired visual sensory perception 
The performance differences between GSKI and WT mice at the shortest stimulus durations could reflect deficits in divided attention or, alternatively, could reflect diminished motivation, locomotor deficits or impaired visual sensory perception. To investigate these alternative possibilities, upon completion of the 5-CSRT task, motivation was tested by subjecting a subset of mice to a progressive ratio (PR) task (Heath et al., 2016). Here, we found that neither the breakpoints (Fig. 4A) nor reward collection latencies (Fig. 4B) differed significantly between genotypes. Consistent with this, we found no differences between genotypes in reward collection latencies in either probe 1 (Fig. 4C) or probe 2 (Fig. 4D) in the 5-CSRT task. Thus, performance deficits of the GSKI mice are not likely attributable to diminished motivation.

We next analyzed locomotor activity within the touch-screen chamber. There were no differences between genotypes in the number of back or front beam-breaks (Figs. 4E, F), suggesting no overt locomotor abnormalities. This conclusion is consistent with prior studies showing that locomotor activity of the GSKI mice during exploration of an arena is similar to that of WT mice (Yue et al., 2015; MatikainenAnkney et al., 2018; Guevara et al., 2020).

Finally, to test visual sensory perception at the shortest stimulus durations of 0.6 and $0.4 \mathrm{sec}$, where deficits in the 5-CSRT task were manifest, we implemented a 1choice version of the task by fixing the stimulus location to the middle response window only, thereby eliminating the requirement that mice divide their attention across 5 spatial locations (Humby et al., 1999). Under the lower attentional demands of this task, GSKI and WT mice showed no significant differences in omissions (Fig. 5A) or in the number of completed trials (Fig. 5B). These data suggest that visual sensory deficits per se are unlikely to underlie the higher percentage of omissions observed in the probe trials. However, there remained a main effect of genotype on correct response latency, with GSKI mice displaying significantly longer latencies (Fig. 5C). Thus, in the 1-choice task, omissions were normalized in the GSKI mice despite persistently slower information processing speed. This suggests that longer response latencies are unlikely to solely underlie the higher rate of omissions observed in the probe trials, though it may exacerbate the burden placed on attentional mechanisms when dividing attention across five screens. 
Cholinergic signaling implicated in attention and processing speed deficits in the GSKI mice

Attentional processes are strongly modulated by central cholinergic mechanisms in rodents and humans (Baxter and Chiba, 1999). We therefore tested whether peripheral administration of the centrally-acting acetylcholinesterase inhibitor donepezil (Kosasa et al., 1999) would mitigate performance deficits displayed by the GSKI mice in the 5-CSRT task. Using a within-subject design, WT and GSKI mice received either saline or donepezil $(0.3 \mathrm{mg} / \mathrm{kg}$, IP), counterbalanced across mice of each genotype, one hour before testing with a $0.4 \mathrm{sec}$ stimulus duration. We found that GSKI mice treated with donepezil made significantly fewer omissions (Fig. 6A) and completed more trials (Fig. 6B) than when treated with saline. Additionally, GSKI mice treated with donepezil showed shorter correct-response latencies in comparison with their performance when treated with saline (Fig. 6C). The GSKI mice displayed no significant differences in numbers of correct responses when treated with donepezil compared to when they were treated with saline (Fig. 6D). In contrast, in WT mice, there were no significant differences in any performance measure between saline and donepezil treatment (Figs. 6E-H). Together, these data suggest that acetylcholinesterase inhibition mitigated performance deficits displayed by the GSKI mice in the 5-CSRT task.

\section{GSKI mice display altered cholinergic innervation of mPFC and striatum}

To understand better a potential basis for the restorative effect of donepezil on attention and processing speed deficits in the young adult GSKI mice, we analyzed the density, size and intensity of immunofluorescent vesicular acetylcholine transporter (VAChT) labeling of cholinergic axons innervating mPFC (areas PL/IL) and dorsomedial striatum in GSKI and WT mice. In both structures, VAChT immunolabeling appeared as fragments of thin fibers studded with varicosities, similar to previous descriptions (Henny and Jones, 2008). We found significantly lower cholinergic fiber innervation densities and smaller immunofluorescent puncta sizes across all layers of mPFC (Fig. 7A-C) and in dorsomedial striatum (Fig. 7D-F) of GSKI mice in comparison with WT mice. There were no significant genotype differences in VAChT immunofluorescence 
intensity. These data reveal a significant anatomical alteration in cholinergic innervation of two principal interconnected structures that mediate executive function in young adult mutant mice, and suggest a basis for the rescue of some behavioral measures by donepezil.

\section{GSKI mice are impaired in goal-directed learning}

We assayed goal-directed actions using a 4-day instrumental conditioning paradigm that in WT mice produces goal-directed action-outcome associations verified subsequently by sensitivity to outcome devaluation (Shan et al., 2014). Both WT and GSKI mice made a similar number of responses under a continuous reinforcement (CRF) schedule on day 1 (Fig. 8A). On subsequent days 2-4 of the instrumental task, the reward was delivered on a random interval (RI) schedule of 15,30 and $30 \mathrm{sec}$. As expected, WT mice made progressively greater numbers of responses on days 2-4 (Fig. 8A) and, following the 4-day instrumental conditioning paradigm, they exhibited sensitivity to reward devaluation (Fig. 8B), indicating goal-directed learning as expected (Shan et al., 2014). In contrast, GSKI mice made significantly fewer responses to the stimulus under the RI schedules on days 3 and 4 in comparison with WT mice (Fig. 8A), and were insensitive to outcome devaluation (Fig. 8B), suggesting an impairment in instrumental conditioning and goal-directed learning.

Goal-directed action-outcome association is also sensitive to motivation (Heath et al., 2016). Thus, following the last outcome devaluation test, we subjected the WT and GSKI mice to a PR task to compare their motivation for the reward. As before, we found no significant differences between genotypes in break points (Fig. 8C). Additionally, there were no differences between genotypes in average reward collection latencies (Fig. 8D). These data suggest that alterations in motivation for the reward are unlikely to underlie deficits in goal-directed learning in GSKI mice.

\section{Cognitive flexibility is unaffected in GSKI mice}

We assayed cognitive flexibility with a pairwise visual discrimination task followed by reversal learning. During the initial visual discrimination phase, we found no significant differences between genotypes in the number of sessions required to reach 
performance criteria or in percent correct responses (Fig. 9A), in session length (Fig. 9B) or in correction responses (Fig. 9C).

In the subsequent reversal phase, when the stimulus/reward contingency was reversed, GSKI and WT mice reached performance criteria after a similar number of sessions, with similar percentages of correct responses (Fig. 9A) and session lengths (Fig. 9B). WT mice showed no significant difference in the number of corrective responses between the discrimination and reversal phases (Fig. 9C), and while GSKI mice showed a slightly greater number of correction responses in the reversal phase in comparison with their number of correction responses in the discrimination phase, there were no significant genotype x phase interactions (Fig. 9C), suggesting that stimulusreward learning was intact in the GSKI mice.

\section{DISCUSSION}

We used visuospatial touchscreen-based operant tasks to assess executive function in young adult male mice carrying a PD-associated Lrrk2-G2019S knockin mutation. GSKI mice displayed significant deficits in visuospatial attention and longer response latencies, suggesting significantly slower information processing. GSKI mice were also impaired in instrumental conditioning and goal-directed learning, while pairwise visual discrimination and reversal learning--a measure of cognitive flexibility-appeared largely unaffected. In humans, deficits in attention and processing speed are characteristics of early cognitive symptoms of idiopathic and several familial forms of PD, including asymptomatic LRRK2-G2019S carriers (Thaler et al., 2012; Robbins and Cools, 2014; Perugini et al., 2018; Piredda et al., 2020). We found that measures of impaired attention in the GSKI mice were rescued by donepezil, a centrally-acting acetylcholinesterase inhibitor, and that cholinergic innervation of mPFC and striatum was significantly sparser than WT, suggesting that altered cholinergic anatomy in brain may have direct or indirect contributions to cognitive dysfunction in young adult GSKI mice. Taken together, these data suggest a framework for better understanding deficient cognitive control mechanisms that may underlie early PD-associated nonmotor cognitive impairment. 
The 5-CSRT task is a sensitive test of visuospatial attention in rodents (Robbins, 2002; Amitai and Markou, 2010). In the GSKI mice, the main hallmark of an attention deficit was a consistently higher percentage of omissions, evident during the training phase but significantly affecting performance at the shortest stimulus durations in the two probe trials when attentional demand is greatest. Control experiments ruled out motivational, locomotor or visual sensory perceptual deficits, thus suggesting a general deficit in divided attention in the GSKI mice. The significantly longer response latencies in probe 1 suggest that GSKI mice have slower information/decision processing speed compared to WT mice, which likely contributed to the significantly greater percentage of omissions. In contrast, response accuracy in the GSKI mice was not significantly different than WT mice. The combination of slower processing speed but preserved accuracy in the mutant mice is a performance profile similar to that described in a study of human non-PD manifesting carriers of the LRRK2-G2019S mutation on the Stroop Color-Word test, where significant performance differences were found in comparison with healthy controls (Thaler et al., 2012). Additionally, although the underlying circuitry involved in such deficits in the mice is currently unknown, generally each of the tasks that we used test cognitive domains that require intact connectivity between areas of medial and orbital prefrontal cortex and dorsomedial striatum (Chudasama and Robbins, 2004; Yin et al., 2005; Izquierdo et al., 2017). In human non-PD manifesting LRRK2-G2019S carriers, fMRI imaging during performance in the Stroop Color-Word test indicated abnormal connectivity between frontal and parietal cortical regions and striatum (Thaler et al., 2013; Bregman et al., 2017), which together suggest some similarities in neural mechanisms and circuits underlying aspects of executive dysfunction in mice and humans.

Some of the various behavioral deficits in the GSKI mice may reflect dysfunction in action selection, which could include faulty behavioral inhibition and/or faulty selection of an appropriate response (Aron, 2007; Friedman and Robbins, 2021). In addition to omissions, GSKI mice made significantly fewer premature responses in the 5-CSRT task (a heightened withholding of responses). While the precise neural basis for such deficits is unclear, our finding that pretreating GSKI mice with the acetylcholinesterase inhibitor donepezil--which increases the perisynaptic concentration of acetylcholine 
(Kosasa et al., 1999)--reversed the high rate of omissions and largely normalized response latencies in the 5-CSRT task implicates cholinergic signaling. In human idiopathic PD, cholinergic function in brain is reduced in both non-demented and demented patients, affecting cognition and other non-motor symptoms (Perez-Lloret and Barrantes, 2016). Similarly, PET studies of human LRRK2-mutation carriers, including those with the G2019S mutation, suggest that LRRK2 mutation carriers with or without PD exhibit heightened acetylcholinesterase activity in several cortical and subcortical regions in comparison with idiopathic PD or healthy controls (Liu et al., 2018). This suggests that deficient cholinergic modulation of circuit function can be evident early, before motor symptoms, which could contribute to early cognitive dysfunction. On the other hand, while acetylcholinesterase inhibitors, including donepezil, have been used clinically to ameliorate cognitive decline in demented PD and Alzheimer's patients (Simuni et al., 2009; Sharma, 2019; Armstrong and Okun, 2020), their effectiveness for improving mild cognitive impairment in early-stage PD is equivocal (Mamikonyan et al., 2015; Baik et al., 2021).

We found no effect of donepezil in WT mice, making it unlikely that the drug nonspecifically elevated motivation or arousal. Rather, acetylcholine signaling, particularly in mPFC, modulates visuospatial attention specifically (Baxter and Chiba, 1999; Bloem et al., 2014). Acetylcholine transients across multiple time-scales are detected within mPFC of rodents during performance of visuospatial tasks that place demands on attentional mechanisms (Arnold et al., 2002; Parikh et al., 2007), including the 5-CSRT task (Passetti et al., 2000), and are triggered by cognitive operations that govern the transition from cue detection monitoring to activation of cue-directed responses (Parikh et al., 2007; Howe et al., 2013; Sarter et al., 2014). Such cue-associated acetylcholine transients reflect innervation of mPFC by basal forebrain cholinergic neurons (Ballinger et al., 2016), but extrinsic and intrinsic cholinergic innervation of striatum is also relevant to attentional and other cognitive control mechanisms. Lesions of striatally-projecting brainstem cholinergic neurons impair attention and reduce response latency in a 5CSRT task (Cyr et al., 2015), while lesions of striatal cholinergic interneurons impair cognitive flexibility (Ragozzino et al., 2009; Bradfield et al., 2013; Aoki et al., 2015). One limit of our study is that donepezil was administered systemically, so it is unknown 
which cholinergic system is involved. Future studies will be needed to address this.

Although the reversal of some aberrant behavioral measures by donepezil in the 5-CSRT task implicates cholinergic signaling, the mechanism that links LRRK2 mutation to cholinergic function, either directly or indirectly, remains speculative. Our analyses of cholinergic innervation of mPFC and dorsomedial striatum indicate that in both structures, cholinergic innervation is significantly less dense in mutants, with smaller immunofluorescently-labeled puncta. Whether lower fiber density reflects fewer cholinergic somata or preserved somata each with sparser terminal axonal fields remains to be determined. The presence of overt anatomical abnormalities in VAChT labeling in young adults is striking in that striatal tyrosine hydroxylase levels and labeling appear normal at similar ages in G2019S knockin mice, although striatal dopamine signaling may be altered early (Volta et al., 2017). Cholinergic neurons may be precociously vulnerable to PD pathogenesis compared to DA neurons, perhaps explaining cognitive abnormalities that often precede abundant DA neuron degeneration. Mechanistically, early vulnerability could reflect G2019S-mediated disruption of trophic support of cholinergic neurons. Previous studies have shown that pathogenic LRRK2 mutations disrupt ciliogenesis in cortical and striatal neurons (Dhekne et al., 2018; Lara Ordónez et al., 2019; Khan et al., 2021), interfering with a cilia-dependent, reciprocal molecular signaling loop that neurotrophically maintains the nigrostriatal circuit (Gonzalez-Reyes et al., 2012). In this signaling loop, striatal DA axons secrete Sonic hedgehog (Shh) that is sensed by cilia on striatal cholinergic interneurons. They, in turn, respond to Shh by secreting GDNF, providing trophic support of both DA and striatal cholinergic neurons. Developing and adult basal forebrain cholinergic neurons also synthesize Shh (Reilly et al., 2002) and require NGF-which in mPFC is secreted by interneurons (Biane et al., 2014)--for survival and maintenance of terminal arbors (Lad et al., 2003). In vitro studies show synergistic trophic support of basal forebrain cholinergic neurons in the presence of both Shh and NGF (Reilly et al., 2002). Thus, it is plausible that G2019S-mediated disruption of neuronal cilia in mPFC or striatum progressively disrupts a neurotrophic loop akin to that described above, derailing proper maintenance of cholinergic innervation and function. 
LRRK2-G2019S can also alter properties of presynaptic neurotransmitter release (Beccano-Kelly et al., 2014; Cirnaru et al., 2014; Pan et al., 2017). While the mutation may affect release of acetylcholine, a direct effect is unlikely, since expression levels of LRRK2 are very low in basal forebrain, cholinergic brainstem nuclei and striatal cholinergic interneurons. In contrast, LRRK2 expression is high in cortical neurons and in striatal projection neurons (SPNs) (Taymans et al., 2006; Giesert et al., 2013; West et al., 2014; Gokce et al., 2016), suggesting that functionally abnormal presynaptic input to cholinergic cells from G2019S-expressing cortical neurons alters appropriate top-down regulation of cholinergic cell firing. A final possibility is that convergent cholinergic and glutamatergic inputs interact to mitigate G2019S-driven deficits in glutamatergic synaptic plasticity that have been described in G2019S knockin mice. The stoichiometry of AMPA-type glutamate receptor subunits at excitatory synapses onto SPNs in adult G2019S knockin mice is altered in comparison with WT striatal synapses (MatikainenAnkney et al., 2018; Chen et al., 2020), and in these mice, both direct- and indirectpathway SPNs in dorsomedial striatum are unable to express corticostriatal long-term potentiation (Matikainen-Ankney et al., 2018). Normal bidirectional synaptic plasticity of corticostriatal synapses is associated with the cognitive processes tested here (Lovinger, 2010; Shan et al., 2014; Marquardt et al., 2021), and cholinergic signaling in $\mathrm{mPFC}$, striatum and elsewhere critically regulates the expression of persistent forms of glutamatergic synaptic plasticity (Calabresi et al., 1999; Centonze et al., 2003; Gong and Ford, 2019; Fernández de Sevilla et al., 2021; Cools and Arnsten, 2022). It is therefore plausible that cognitive deficits in the mutants reflect such abnormal glutamatergic synaptic signaling and plasticity in dorsomedial striatum, and that boosting ambient acetylcholine levels by donepezil is sufficient to drive convergent intracellular signaling pathways to compensate for such plasticity deficits. 


\section{References}

Amitai N, Markou A (2010) Disruption of performance in the five-choice serial reaction time task induced by administration of $\mathrm{N}$-methyl-D-aspartate receptor antagonists: relevance to cognitive dysfunction in schizophrenia. Biol Psychiatry 68:5-16.

Aoki S, Liu AW, Zucca A, Zucca S, Wickens JR (2015) Role of Striatal Cholinergic Interneurons in Set-Shifting in the Rat. J Neurosci 35:9424-9431.

Armstrong MJ, Okun MS (2020) Diagnosis and Treatment of Parkinson Disease: A Review. JAMA 323:548-560.

Arnold HM, Burk JA, Hodgson EM, Sarter M, Bruno JP (2002) Differential cortical acetylcholine release in rats performing a sustained attention task versus behavioral control tasks that do not explicitly tax attention. Neuroscience 114:451-460.

Aron AR (2007) The neural basis of inhibition in cognitive control. Neuroscientist 13:214-228.

Baik K, Kim SM, Jung JH, Lee YH, Chung SJ, Yoo HS, Ye BS, Lee PH, Sohn YH, Kang SW, Kang SY (2021) Donepezil for mild cognitive impairment in Parkinson's disease. Sci Rep 11:4734.

Ballinger EC, Ananth M, Talmage DA, Role LW (2016) Basal Forebrain Cholinergic Circuits and Signaling in Cognition and Cognitive Decline. Neuron 91:1199_ 1218.

Bari A, Dalley JW, Robbins TW (2008) The application of the 5-choice serial reaction time task for the assessment of visual attentional processes and impulse control in rats. Nat Protoc 3:759-767.

Bartko SJ, Vendrell I, Saksida LM, Bussey TJ (2011) A computer-automated touchscreen paired-associates learning (PAL) task for mice: impairments 
following administration of scopolamine or dicyclomine and improvements following donepezil. Psychopharmacology (Berl) 214:537-548.

Bates D, Mächler M, Bolker B, Walker S (2015) Fitting Linear Mixed-Effects Models Using Ime4. Journal of Statistical Software 67:1-48 Available at: https://doi.org/10.18637/jss.v067.i01.

Baxter MG, Chiba AA (1999) Cognitive functions of the basal forebrain. Curr Opin Neurobiol 9:178-183.

Beccano-Kelly DA, Kuhlmann N, Tatarnikov I, Volta M, Munsie LN, Chou P, Cao L-P, Han H, Tapia L, Farrer MJ, Milnerwood AJ (2014) Synaptic function is modulated by LRRK2 and glutamate release is increased in cortical neurons of G2019S LRRK2 knock-in mice. Front Cell Neurosci 8:301.

Ben Romdhan S, Farhat N, Nasri A, Lesage S, Hdiji O, Ben Djebara M, Landoulsi Z, Stevanin G, Brice A, Damak M, Gouider R, Mhiri C (2018) LRRK2 G2019S Parkinson's disease with more benign phenotype than idiopathic. Acta Neurol Scand 138:425-431.

Benson DL, Huntley GW (2019) Are we listening to everything the PARK genes are telling us? J Comp Neurol 527:1527-1540 Available at: https://www.ncbi.nlm.nih.gov/pubmed/30680728.

Biane J, Conner JM, Tuszynski MH (2014) Nerve growth factor is primarily produced by GABAergic neurons of the adult rat cortex. Front Cell Neurosci 8:220.

Bloem B, Poorthuis RB, Mansvelder HD (2014) Cholinergic modulation of the medial prefrontal cortex: the role of nicotinic receptors in attention and regulation of neuronal activity. Front Neural Circuits 8:17.

Bohnen NI, Albin RL, Müller MLTM, Petrou M, Kotagal V, Koeppe RA, Scott PJH, Frey KA (2015) Frequency of cholinergic and caudate nucleus dopaminergic deficits across the predemented cognitive spectrum of Parkinson disease and evidence of interaction effects. JAMA Neurol 72:194-200. 
Bohnen NI, Kaufer DI, Hendrickson R, Ivanco LS, Lopresti BJ, Constantine GM, Mathis CA, Davis JG, Moore RY, Dekosky ST (2006) Cognitive correlates of cortical cholinergic denervation in Parkinson's disease and parkinsonian dementia. J Neurol 253:242-247.

Bolam JP, Wainer BH, Smith AD (1984) Characterization of cholinergic neurons in the rat neostriatum. A combination of choline acetyltransferase immunocytochemistry, Golgi-impregnation and electron microscopy. Neuroscience 12:711-718.

Bradfield LA, Bertran-Gonzalez J, Chieng B, Balleine BW (2013) The thalamostriatal pathway and cholinergic control of goal-directed action: interlacing new with existing learning in the striatum. Neuron 79:153-166.

Bregman N, Thaler A, Mirelman A, Helmich RC, Gurevich T, Orr-Urtreger A, Marder K, Bressman S, Bloem BR, Giladi N, LRRK2 Ashkenazi Jewish consortium (2017) A cognitive fMRI study in non-manifesting LRRK2 and GBA carriers. Brain Struct Funct 222:1207-1218.

Bussey TJ, Muir JL, Everitt BJ, Robbins TW (1997) Triple dissociation of anterior cingulate, posterior cingulate, and medial frontal cortices on visual discrimination tasks using a touchscreen testing procedure for the rat. Behav Neurosci 111:920-936.

Calabresi P, Centonze D, Gubellini P, Bernardi G (1999) Activation of M1-like muscarinic receptors is required for the induction of corticostriatal LTP. Neuropharmacology 38:323-326.

Cammisuli DM, Cammisuli SM, Fusi J, Franzoni F, Pruneti C (2019) Parkinson's Disease-Mild Cognitive Impairment (PD-MCI): A Useful Summary of Update Knowledge. Front Aging Neurosci 11:303. 
Centonze D, Gubellini P, Pisani A, Bernardi G, Calabresi P (2003) Dopamine, acetylcholine and nitric oxide systems interact to induce corticostriatal synaptic plasticity. Rev Neurosci 14:207-216.

Chen C, Soto G, Dumrongprechachan V, Bannon N, Kang S, Kozorovitskiy Y, Parisiadou L (2020) Pathway-specific dysregulation of striatal excitatory synapses by LRRK2 mutations. Elife 9.

Choi SH, Jung TM, Lee JE, Lee S-K, Sohn YH, Lee PH (2012) Volumetric analysis of the substantia innominata in patients with Parkinson's disease according to cognitive status. Neurobiol Aging 33:1265-1272.

Chudasama Y, Robbins TW (2004) Psychopharmacological approaches to modulating attention in the five-choice serial reaction time task: implications for schizophrenia. Psychopharmacology (Berl) 174:86-98.

Cirnaru MD, Marte A, Belluzzi E, Russo I, Gabrielli M, Longo F, Arcuri L, Murru L, Bubacco L, Matteoli M, Fedele E, Sala C, Passafaro M, Morari M, Greggio E, Onofri F, Piccoli G (2014) LRRK2 kinase activity regulates synaptic vesicle trafficking and neurotransmitter release through modulation of LRRK2 macromolecular complex. Front Mol Neurosci 7:49.

Cools R, Arnsten AFT (2022) Neuromodulation of prefrontal cortex cognitive function in primates: the powerful roles of monoamines and acetylcholine. Neuropsychopharmacology 47:309-328.

Cyr M, Parent MJ, Mechawar N, Rosa-Neto P, Soucy J-P, Clark SD, Aghourian M, Bedard M-A (2015) Deficit in sustained attention following selective cholinergic lesion of the pedunculopontine tegmental nucleus in rat, as measured with both post-mortem immunocytochemistry and in vivo PET imaging with $\left[{ }^{18} \mathrm{~F}\right]$ fluoroethoxybenzovesamicol. Behav Brain Res 278:107-114. 
Dautan D, Hacioğlu Bay H, Bolam JP, Gerdjikov TV, Mena-Segovia J (2016) Extrinsic Sources of Cholinergic Innervation of the Striatal Complex: A Whole-Brain Mapping Analysis. Front Neuroanat 10:1.

Dawson TM, Ko HS, Dawson VL (2010) Genetic animal models of Parkinson's disease. Neuron 66:646-661.

Dhekne HS, Yanatori I, Gomez RC, Tonelli F, Diez F, Schüle B, Steger M, Alessi DR, Pfeffer SR (2018) A pathway for Parkinson's Disease LRRK2 kinase to block primary cilia and Sonic hedgehog signaling in the brain. Elife 7:e40202.

Dougherty SE, Kajstura TJ, Jin Y, Chan-Cortés MH, Kota A, Linden DJ (2020) Catecholaminergic axons in the neocortex of adult mice regrow following brain injury. Exp Neurol 323:113089.

Elbaz A, Carcaillon L, Kab S, Moisan F (2016) Epidemiology of Parkinson's disease. Rev Neurol (Paris) 172:14-26.

Everitt BJ, Robbins TW (1997) Central cholinergic systems and cognition. Annu Rev Psychol 48:649-684.

Fernández de Sevilla D, Núñez A, Buño W (2021) Muscarinic Receptors, from Synaptic Plasticity to its Role in Network Activity. Neuroscience 456:60-70.

Friedman NP, Robbins TW (2021) The role of prefrontal cortex in cognitive control and executive function. Neuropsychopharmacology.

Gaig C, Vilas D, Infante J, Sierra M, García-Gorostiaga I, Buongiorno M, Ezquerra M, Martí MJ, Valldeoriola F, Aguilar M, Calopa M, Hernandez-Vara J, Tolosa E (2014) Nonmotor symptoms in LRRK2 G2019S associated Parkinson's disease. PLoS One 9:e108982.

Giesert F, Hofmann A, Bürger A, Zerle J, Kloos K, Hafen U, Ernst L, Zhang J, VogtWeisenhorn DM, Wurst W (2013) Expression Analysis of Lrrk1, Lrrk2 and Lrrk2 
Splice Variants in Mice Chu CT, ed. PLoS ONE 8:e63778 Available at: https://dx.plos.org/10.1371/journal.pone.0063778 [Accessed May 26, 2021].

Gokce O, Stanley GM, Treutlein B, Neff NF, Camp JG, Malenka RC, Rothwell PE, Fuccillo MV, Südhof TC, Quake SR (2016) Cellular Taxonomy of the Mouse Striatum as Revealed by Single-Cell RNA-Seq. Cell Rep 16:1126-1137.

Gong S, Ford CP (2019) Cholinergic Interneurons Provide a Link to Balance Excitation across Striatal Output Neurons. Neuron 103:351-353.

Gonzalez-Reyes LE, Verbitsky M, Blesa J, Jackson-Lewis V, Paredes D, Tillack K, Phani S, Kramer ER, Przedborski S, Kottmann AH (2012) Sonic hedgehog maintains cellular and neurochemical homeostasis in the adult nigrostriatal circuit. Neuron 75:306-319.

Guevara CA, Matikainen-Ankney BA, Kezunovic N, LeClair K, Conway AP, Menard C, Flanigan ME, Pfau M, Russo SJ, Benson DL, Huntley GW (2020) LRRK2 mutation alters behavioral, synaptic, and nonsynaptic adaptations to acute social stress. J Neurophysiol 123:2382-2389.

Healy DG et al. (2008) Phenotype, genotype, and worldwide genetic penetrance of LRRK2-associated Parkinson's disease: a case-control study. Lancet Neurol 7:583-590 Available at: http://www.ncbi.nlm.nih.gov/entrez/query.fcgi?cmd=Retrieve\&db=PubMed\&dopt= Citation\&list_uids $=18539534$.

Heath CJ, Phillips BU, Bussey TJ, Saksida LM (2016) Measuring Motivation and Reward-Related Decision Making in the Rodent Operant Touchscreen System. Curr Protoc Neurosci 74:8.34.1-8.34.20.

Helmich RC, Thaler A, van Nuenen BFL, Gurevich T, Mirelman A, Marder KS, Bressman S, Orr-Urtreger A, Giladi N, Bloem BR, Toni I, LRRK2 Ashkenazi Jewish Consortium (2015) Reorganization of corticostriatal circuits in healthy G2019S LRRK2 carriers. Neurology 84:399-406. 
Henny P, Jones BE (2008) Projections from basal forebrain to prefrontal cortex comprise cholinergic, GABAergic and glutamatergic inputs to pyramidal cells or interneurons. Eur J Neurosci 27:654-670.

Howe WM, Berry AS, Francois J, Gilmour G, Carp JM, Tricklebank M, Lustig C, Sarter $M$ (2013) Prefrontal cholinergic mechanisms instigating shifts from monitoring for cues to cue-guided performance: converging electrochemical and fMRI evidence from rats and humans. J Neurosci 33:8742-8752.

Humby T, Laird FM, Davies W, Wilkinson LS (1999) Visuospatial attentional functioning in mice: interactions between cholinergic manipulations and genotype. Eur $\mathrm{J}$ Neurosci 11:2813-2823.

Hussein A, Guevara CA, Del Valle P, Gupta S, Benson DL, Huntley GW (2021) NonMotor Symptoms of Parkinson's Disease: The Neurobiology of Early Psychiatric and Cognitive Dysfunction. Neuroscientist:10738584211011980.

Izquierdo A, Brigman JL, Radke AK, Rudebeck PH, Holmes A (2017) The neural basis of reversal learning: An updated perspective. Neuroscience 345:12-26.

Khan SS, Sobu Y, Dhekne HS, Tonelli F, Berndsen K, Alessi DR, Pfeffer SR (2021) Pathogenic LRRK2 control of primary cilia and Hedgehog signaling in neurons and astrocytes of mouse brain. Elife 10:e67900.

Kim I, Shin N-Y, Yunjin Bak null, Hyu Lee P, Lee S-K, Mee Lim S (2017) Early-onset mild cognitive impairment in Parkinson's disease: Altered corticopetal cholinergic network. Sci Rep 7:2381.

Kosasa T, Kuriya Y, Matsui K, Yamanishi Y (1999) Effect of donepezil hydrochloride (E2020) on basal concentration of extracellular acetylcholine in the hippocampus of rats. Eur J Pharmacol 380:101-107.

Kuznetsova A, Brockhoff PB, Christensen RHB (2017) ImerTest Package: Tests in Linear Mixed Effects Models. Journal of Statistical Software 82:1-26 Available at: https://doi.org/10.18637/jss.v082.i13. 
Lad SP, Neet KE, Mufson EJ (2003) Nerve growth factor: structure, function and therapeutic implications for Alzheimer's disease. Curr Drug Targets CNS Neurol Disord 2:315-334.

Lara Ordónez AJ, Fernández B, Fdez E, Romo-Lozano M, Madero-Pérez J, Lobbestael E, Baekelandt V, Aiastui A, López de Munaín A, Melrose HL, Civiero L, Hilfiker S (2019) RAB8, RAB10 and RILPL1 contribute to both LRRK2 kinase-mediated centrosomal cohesion and ciliogenesis deficits. Hum Mol Genet 28:3552-3568.

Lenth RV (2022). emmeans: Estimated Marginal Means, aka Least-Squares Means. R package version 1.7.2. https://CRAN.R-project.org/package=emmeans

Lewis SJG, Dove A, Robbins TW, Barker RA, Owen AM (2003) Cognitive impairments in early Parkinson's disease are accompanied by reductions in activity in frontostriatal neural circuitry. J Neurosci 23:6351-6356.

Liu S-Y et al. (2018) The effect of LRRK2 mutations on the cholinergic system in manifest and premanifest stages of Parkinson's disease: a cross-sectional PET study. Lancet Neurol 17:309-316.

Lovinger DM (2010) Neurotransmitter roles in synaptic modulation, plasticity and learning in the dorsal striatum. Neuropharmacology 58:951-961.

Mamikonyan E, Xie SX, Melvin E, Weintraub D (2015) Rivastigmine for mild cognitive impairment in Parkinson disease: a placebo-controlled study. Mov Disord 30:912-918.

Mar AC, Horner AE, Nilsson SRO, Alsiö J, Kent BA, Kim CH, Holmes A, Saksida LM, Bussey TJ (2013) The touchscreen operant platform for assessing executive function in rats and mice. Nat Protoc 8:1985-2005.

Marquardt K, Josey M, Kenton JA, Cavanagh JF, Holmes A, Brigman JL (2021) Impaired cognitive flexibility following NMDAR-GluN2B deletion is associated with altered orbitofrontal-striatal function. Neuroscience 475:230-245. 
Martin I, Kim JW, Dawson VL, Dawson TM (2014) LRRK2 pathobiology in Parkinson's disease. J Neurochem 131:554-565.

Matikainen-Ankney BA, Kezunovic N, Menard C, Flanigan ME, Zhong Y, Russo SJ, Benson DL, Huntley GW (2018) Parkinson's Disease-Linked LRRK2-G2019S Mutation Alters Synaptic Plasticity and Promotes Resilience to Chronic Social Stress in Young Adulthood. J Neurosci 38:9700-9711.

Matikainen-Ankney BA, Kezunovic N, Mesias RE, Tian Y, Williams FM, Huntley GW, Benson DL (2016) Altered Development of Synapse Structure and Function in Striatum Caused by Parkinson's Disease-Linked LRRK2-G2019S Mutation. J Neurosci 36:7128-7141.

Mirelman A, Alcalay RN, Saunders-Pullman R, Yasinovsky K, Thaler A, Gurevich T, Mejia-Santana H, Raymond D, Gana-Weisz M, Bar-Shira A, Ozelius L, Clark L, Orr-Urtreger A, Bressman S, Marder K, Giladi N, LRRK2 AJ consortium (2015) Nonmotor symptoms in healthy Ashkenazi Jewish carriers of the G2019S mutation in the LRRK2 gene. Mov Disord 30:981-986.

Muslimovic D, Post B, Speelman JD, Schmand B (2005) Cognitive profile of patients with newly diagnosed Parkinson disease. Neurology 65:1239-1245.

Obermayer J, Luchicchi A, Heistek TS, de Kloet SF, Terra H, Bruinsma B, Mnie-Filali O, Kortleven C, Galakhova AA, Khalil AJ, Kroon T, Jonker AJ, de Haan R, van de Berg WDJ, Goriounova NA, de Kock CPJ, Pattij T, Mansvelder HD (2019) Prefrontal cortical ChAT-VIP interneurons provide local excitation by cholinergic synaptic transmission and control attention. Nat Commun 10:5280.

Paisan-Ruiz C, Lewis PA, Singleton AB (2013) LRRK2: cause, risk, and mechanism. J Parkinsons Dis 3:85-103 Available at: http://www.ncbi.nlm.nih.gov/pubmed/23938341.

Paisán-Ruiz C, Lewis PA, Singleton AB (2013) LRRK2: cause, risk, and mechanism. J Parkinsons Dis 3:85-103. 
Pan P-Y, Li X, Wang J, Powell J, Wang Q, Zhang Y, Chen Z, Wicinski B, Hof P, Ryan TA, Yue Z (2017) Parkinson's Disease-Associated LRRK2 Hyperactive Kinase Mutant Disrupts Synaptic Vesicle Trafficking in Ventral Midbrain Neurons. J Neurosci 37:11366-11376.

Parikh V, Kozak R, Martinez V, Sarter M (2007) Prefrontal acetylcholine release controls cue detection on multiple timescales. Neuron 56:141-154.

Pasquini J, Brooks DJ, Pavese N (2021) The Cholinergic Brain in Parkinson's Disease. Mov Disord Clin Pract 8:1012-1026.

Passetti F, Dalley JW, O’Connell MT, Everitt BJ, Robbins TW (2000) Increased acetylcholine release in the rat medial prefrontal cortex during performance of a visual attentional task. Eur J Neurosci 12:3051-3058.

Perez-Lloret S, Barrantes FJ (2016) Deficits in cholinergic neurotransmission and their clinical correlates in Parkinson's disease. NPJ Parkinsons Dis 2:16001.

Perugini A, Ditterich J, Shaikh AG, Knowlton BJ, Basso MA (2018) Paradoxical Decision-Making: A Framework for Understanding Cognition in Parkinson's Disease. Trends Neurosci 41:512-525.

Piredda R, Desmarais P, Masellis M, Gasca-Salas C (2020) Cognitive and psychiatric symptoms in genetically determined Parkinson's disease: a systematic review. Eur J Neurol 27:229-234.

Prakash KM, Nadkarni NV, Lye W-K, Yong M-H, Tan E-K (2016) The impact of nonmotor symptoms on the quality of life of Parkinson's disease patients: a longitudinal study. Eur J Neurol 23:854-860.

R Core Team (2021). R: A language and environment for statistical computing. $R$ Foundation for Statistical Computing, Vienna, Austria. URL https://www.Rproject.org/. 
Ragozzino ME (2007) The contribution of the medial prefrontal cortex, orbitofrontal cortex, and dorsomedial striatum to behavioral flexibility. Ann N Y Acad Sci 1121:355-375.

Ragozzino ME, Mohler EG, Prior M, Palencia CA, Rozman S (2009) Acetylcholine activity in selective striatal regions supports behavioral flexibility. Neurobiol Learn Mem 91:13-22.

Reekes TH, Higginson Cl, Ledbetter CR, Sathivadivel N, Zweig RM, Disbrow EA (2020) Sex specific cognitive differences in Parkinson disease. NPJ Parkinsons Dis 6:7.

Reilly JO, Karavanova ID, Williams KP, Mahanthappa NK, Allendoerfer KL (2002) Cooperative effects of Sonic Hedgehog and NGF on basal forebrain cholinergic neurons. Mol Cell Neurosci 19:88-96.

Robbins TW (2002) The 5-choice serial reaction time task: behavioural pharmacology and functional neurochemistry. Psychopharmacology (Berl) 163:362-380.

Robbins TW, Cools R (2014) Cognitive deficits in Parkinson's disease: a cognitive neuroscience perspective. Mov Disord 29:597-607.

Romberg C, Mattson MP, Mughal MR, Bussey TJ, Saksida LM (2011) Impaired attention in the 3xTgAD mouse model of Alzheimer's disease: rescue by donepezil (Aricept). J Neurosci 31:3500-3507.

Sarter M, Lustig C, Howe WM, Gritton H, Berry AS (2014) Deterministic functions of cortical acetylcholine. Eur J Neurosci 39:1912-1920.

Selemon LD, Goldman-Rakic PS (1988) Common cortical and subcortical targets of the dorsolateral prefrontal and posterior parietal cortices in the rhesus monkey: evidence for a distributed neural network subserving spatially guided behavior. $J$ Neurosci 8:4049-4068. 
Shan Q, Ge M, Christie MJ, Balleine BW (2014) The acquisition of goal-directed actions generates opposing plasticity in direct and indirect pathways in dorsomedial striatum. J Neurosci 34:9196-9201.

Sharma K (2019) Cholinesterase inhibitors as Alzheimer's therapeutics (Review). Mol Med Rep 20:1479-1487.

Simuni T, Lyons KE, Pahwa R, Hauser RA, Comella C, Elmer L, Weintraub D (2009) Treatment of early Parkinson's disease. Part 2. Eur Neurol 61:206-215.

Taymans J-M, Van den Haute C, Baekelandt V (2006) Distribution of PINK1 and LRRK2 in rat and mouse brain. J Neurochem 98:951-961.

Thaler A, Mirelman A, Gurevich T, Simon E, Orr-Urtreger A, Marder K, Bressman S, Giladi N, LRRK2 Ashkenazi Jewish Consortium (2012) Lower cognitive performance in healthy G2019S LRRK2 mutation carriers. Neurology 79:10271032.

Thaler A, Mirelman A, Helmich RC, van Nuenen BFL, Rosenberg-Katz K, Gurevich T, Orr-Urtreger A, Marder K, Bressman S, Bloem BR, Giladi N, Hendler T, LRRK2 Ashkenazi Jewish consortium (2013) Neural correlates of executive functions in healthy G2019S LRRK2 mutation carriers. Cortex 49:2501-2511.

Volta M, Beccano-Kelly DA, Paschall SA, Cataldi S, Maclsaac SE, Kuhlmann N, Kadgien CA, Tatarnikov I, Fox J, Khinda J, Mitchell E, Bergeron S, Melrose H, Farrer MJ, Milnerwood AJ (2017) Initial elevations in glutamate and dopamine neurotransmission decline with age, as does exploratory behavior, in LRRK2 G2019S knock-in mice. eLife 6:e28377 Available at: https://elifesciences.org/articles/28377 [Accessed May 26, 2021].

West AB, Cowell RM, Daher JPL, Moehle MS, Hinkle KM, Melrose HL, Standaert DG, Volpicelli-Daley LA (2014) Differential LRRK2 expression in the cortex, striatum, and substantia nigra in transgenic and nontransgenic rodents: LRRK2 distribution 
in the nigrostriatal pathway. J Comp Neurol 522:2465-2480 Available at: http://doi.wiley.com/10.1002/cne.23583 [Accessed May 26, 2021].

Wolters AF, van de Weijer SCF, Leentjens AFG, Duits AA, Jacobs HIL, Kuijf ML (2019) Resting-state fMRI in Parkinson's disease patients with cognitive impairment: A meta-analysis. Parkinsonism Relat Disord 62:16-27.

Yin HH, Ostlund SB, Knowlton BJ, Balleine BW (2005) The role of the dorsomedial striatum in instrumental conditioning. Eur J Neurosci 22:513-523.

Yue $\mathrm{M}$ et al. (2015) Progressive dopaminergic alterations and mitochondrial abnormalities in LRRK2 G2019S knock-in mice. Neurobiol Dis 78:172-195 Available at: http://www.ncbi.nlm.nih.gov/pubmed/25836420.

Yuede CM, Dong H, Csernansky JG (2007) Anti-dementia drugs and hippocampaldependent memory in rodents. Behav Pharmacol 18:347-363.

Zimprich A et al. (2004) Mutations in LRRK2 cause autosomal-dominant parkinsonism with pleomorphic pathology. Neuron 44:601-607. 


\section{Figure Legends}

Figure 1. Training performance of WT and GSKI mice in the 5-CSRT task. A, WT (black lines) and GSKI (blue lines) showed similar percentages of correct responses across all stimulus durations [main effect (genotype): $F_{1,35}=0.195, p=0.66$ ]. $B$, GSKI mice made significantly more omissions [main effect (genotype): $F_{1,35}=11.26$, $p=0.0019$; genotype $x$ stimulus duration, $F_{4,510}=2.72, p=0.029$; comparisons of estimated marginal means, $8 \mathrm{sec}, p=.0035 ; 4 \mathrm{sec}, p=0.012 ; 2 \mathrm{sec}, p=0.0001]$. C, GSKI mice showed significantly greater accuracy in comparison with WT mice [main effect (genotype): $F_{1,35}=8.62, p=0.005$, but no genotype $x$ stimulus duration interaction, $\left.F_{4,510}=1.58, p=0.18\right]$. $D, G S K I$ mice (blue bars) completed fewer trials compared to WT mice (grey bars) [main effect (genotype): $F_{1,35}=5.78, p=0.022$, but no genotype $x$ stimulus duration interaction, $F_{4,510}=1.92, p=0.11$. E, GSKI mice showed significantly longer correct response latencies in comparison with WT mice [main effect (genotype): $F_{1,35}=5.94, p=0.02$; genotype $x$ stimulus duration interaction, $F_{4,510}=7.43, p<.0001$; comparisons of estimated marginal means, 32 sec, $p<0.0001 ; 16 \mathrm{sec}, \mathrm{p}=0.0005]$. F, GSKI mice made significantly fewer premature responses in comparison with WT mice [main effect (genotype): $F_{1,35}=$ 8.38, $p=0.0065$, but no genotype $x$ stimulus duration interaction, $F_{4,510}=0.67, p=$ 0.61]. $\mathrm{n}=19 \mathrm{WT}, 18 \mathrm{GSKI}$ mice.

Figure 2. Performance of WT and GSKI mice on probe trial 1 in the 5-CSRT task. A, GSKI mice (blue lines) showed a greater percentage of omissions in comparison with WT mice (black lines) [main effect (genotype): $F_{1, ~ 42.9}=36.3, p<0.0001$, stimulus duration $x$ genotype: $F_{5, \sim 676.7}=9.72, p<0.0001$; comparisons of estimated marginal means, $1.5 \mathrm{sec}, \mathrm{p}=.014 ; 0.8 \mathrm{sec}, \mathrm{p}=0.0002 ; 0.6$ and $0.4 \mathrm{sec}, \mathrm{p}<0.0001]$. B, GSKI mice showed a decrease in percentage of correct responses at the shortest stimulus duration [main effect (genotype): $F_{1,40.1}=17.4, p=0.0002$, stimulus duration $x$ genotype: $F_{5, \sim 676.4}=8.06, p<0.0001$; comparisons of estimated marginal means, 1.5 sec, $p=0.047 ; 0.8 \mathrm{sec}, p=0.0002 ; 0.6 \mathrm{sec}, p=0.0001,0.4 \mathrm{sec}, p<0.0001]$. C, WT and GSKI showed similar accuracy across all stimulus durations except the shortest 
$(0.4 \mathrm{sec})$ [main effect (genotype): $F_{1, \sim 42.5}=1.33, p=0.25$, stimulus duration $x$ genotype: $F_{5, \sim 677}=5.59, p<0.0001$; comparisons of estimated marginal means, $0.4 \mathrm{sec}, p<$ 0.0001]. D, GSKI mice (blue bars) completed fewer trials in comparison with WT mice (grey bars) [main effect (genotype): $F_{1, \sim 41.7}=31.7, p<0.0001$, stimulus duration $x$ genotype: $F_{5, \sim 676.7}=8.94, p<0.0001$; comparisons of estimated marginal means, 1.5 sec, $p=0.01 ; 0.8 \mathrm{sec}, p=0.0001 ; 0.6$ and $0.4 \mathrm{sec}, p<0.0001]$. E, GSKI mice showed significantly longer correct response latencies at shorter stimulus durations [main effect (genotype): $F_{1, \sim 39.3}=5.37, p=0.026$, stimulus duration $x$ genotype: $F_{5, \sim 676.9}=4.70, p=$ 0.0003; comparisons of estimated marginal means, $0.6 \mathrm{sec}, \mathrm{p}=0.0008 ; 0.4 \mathrm{sec}, \mathrm{p}=$ 0.0001]. F, GSKI mice made significantly fewer premature responses during the ITI [main effect (genotype): $\mathrm{F}_{1, \sim 44.5}=8.99, \mathrm{p}=0.0044$ ]. $\mathrm{n}=19 \mathrm{WT}, 17 \mathrm{GSKI}$ mice.

Figure 3. Performance of WT and GSKI mice on probe trial 2 in the 5-CSRT task. A, GSKI mice (blue line) displayed significantly more omissions at the shortest stimulus duration in comparison with WT mice (black line) [stimulus duration $x$ genotype: $\mathrm{F}_{3,246}=$ 8.47, $p<0.0001$; comparisons of estimated marginal means, $0.6 \mathrm{sec}, \mathrm{p}=0.0001$ ]. $\mathbf{B}$, GSKI mice showed a decreased percentage of correct responses at the shortest stimulus duration [stimulus duration x genotype: $F_{3,246}=4.50, p=0.0043$; comparisons of estimated marginal means, $0.6 \mathrm{sec}, \mathrm{p}=0.0001]$. C, GSKI and WT mice showed similar accuracy [main effect(genotype): $F_{1,34}=0.052, p=0.82$; stimulus duration $x$ genotype: $\left.F_{3,246}=0.25, p=0.86\right]$. D, GSKI mice (blue bars) showed similar correct response latencies in comparison with WT mice (grey bars) [main effect (genotype): $F_{1,34}=3.38, p=0.075$; stimulus duration $x$ genotype: $\left.F_{3,246}=0.73, p=0.54\right]$. $E$, There were no significant differences between genotypes in premature responses [main effect (genotype): $F_{1,34}=0.024, p=0.88$; stimulus duration $x$ genotype: $F_{3,246}=0.066, p=$ 0.98 ] nor in (F) perseverative responses [main effect (genotype): $F_{1,34}=3.88, p=0.057$; stimulus duration $x$ genotype: $\left.F_{3,246}=1.20, p=0.31\right] . n=13$ WT, 11 GSKI mice.

Figure 4. No differences between genotypes in motivation for reward-related behaviors or locomotor activity. In a progressive ratio (PR4) task to test for motivation, the breakpoints $(\mathbf{A})$ and reward collection latencies $(\mathbf{B})$ were similar between genotypes 
(breakpoints: Welch's t-test, $\mathrm{t}_{18.87}=0.166, \mathrm{p}=0.87$; latencies: Welch's t-test, $\mathrm{t}_{21.93}=$ 1.23, $p=0.23)$. Similarly, there were no differences between genotypes in reward collection latencies in probe 1 [C, main effect (genotype): $F_{1, \sim 42.3}=0.046, p=0.83$ ] or probe $2\left(D\right.$, Welch's t-test, $\left.t_{31.7}=1.37, P=0.18\right)$ in the 5-CSRT task $(n=13$ WT, 11 GSKI mice) in the 5-CSRT. There were no differences between genotypes in numbers of back beam breaks $\left(E\right.$, Welch's t-test, $\left.t_{19.73}=0.73, p=0.48\right)$ or front beam breaks $(\mathbf{F}$, Welch's t-test, $\mathrm{t}_{21.76}=0.53, \mathrm{p}=0.6$ ) during the progressive ratio task, suggesting no differences in locomotion. For the progressive ratio task, $\mathrm{n}=13 \mathrm{WT}, 11 \mathrm{GSKI}$ mice.

Figure 5. Reducing attentional demand in a 1-choice version of the serial reaction time task normalizes performance deficits in GSKI mice observed at the shortest stimulus durations. There were no differences between genotypes in percent omissions [main effect (genotype): $F_{1,20}=0.0304, p=0.86$; stimulus duration $x$ genotype: $F_{2,106}=0.40, p=0.67$ ] or in number of completed trials [main effect (genotype): $F_{1,20}=0.0002, p=0.99$ stimulus duration $x$ genotype: $F_{2,106}=0.35, p=$ $0.70]$ in the 1-choice task. There remained a main effect of genotype on correct response latency $(\mathbf{C})$ similar to the 5-CSRT, with GSKI mice showing significantly longer response latencies [main effect (genotype): $F_{1,20}=5.24, p=0.033$ ]. $n=13$ WT, 9 GSKI mice.

Figure 6. Systemic administration of donepezil, an acetylcholinesterase inhibitor, ameliorated performance deficits of the GSKI mice in the 5-CSRT task. Donepezil (0.3 mg/kg, IP) or saline was administered to GSKI mice (blue lines, A-D) or WT mice (grey lines, E-H), using a within-subject design, $1 \mathrm{hr}$ before testing in the 5CSRT task with a $0.4 \mathrm{sec}$ stimulus duration. Compared to saline treatment (open circles), GSKI mice treated with donepezil (black circles) made significantly fewer omissions (A, genotype $x$ treatment: $F_{1,64}=5.72, p=0.020$; comparisons of estimated marginal means, GSKI saline vs donepezil, $p=0.003$; $\mathbf{E}$, WT saline vs donepezil, $p=0.97$ ) and showed an increased number of completed trials (B, genotype $x$ treatment: $F_{1,64}=6.47, p=0.013$; comparisons of estimated marginal 
means, GSKI saline vs donepezil, $p=0.0016$; F, WT saline vs donepezil, $p=0.98$ ). Treating GSKI mice with donepezil lowered correct response latencies compared to saline treatment $\left(C\right.$, genotype $x$ treatment: $F_{1,64}=5.91, p=0.018$; comparisons of estimated marginal means, GSKI saline vs donepezil, $p=0.016$; F, WT saline vs donepezil, $p=0.41)$. There was no effect of donepezil on percent correct responses ( $D$, genotype $x$ treatment: $\left.F_{1,64}=2.15, p=0.15\right)$. In contrast to GSKI mice, donepezil treatment in WT mice had no significant effects (ns) on omissions $(\mathbf{E})$, number of completed trials $(\mathbf{F})$, response latencies $(\mathbf{G})$ or percent correct responses $(\mathbf{H})$ in comparison with saline treatment. $n=13$ WT, 9 GSKI mice.

Figure 7. GSKI mice show altered pattern of cholinergic fiber innervation in mPFC and dorsomedial striatum in comparison with WT mice. Representative confocal microscope images of VAChT immunofluorescent labeling of cholinergic fibers in L5 of mPFC areas PL/IL (A, B) or dorsomedial striatum (D, E) in WT (left images of pair) or GSKI mice (right images of pair), as indicated. bars $=50 \mu \mathrm{m} . \mathbf{C}, \mathbf{F}$, Box-plots show that in PL/IL (C) and in dorsomedial striatum (F), fiber innervation density is significantly sparser and VAChT-labeled puncta size is significantly smaller in GSKI mice in comparison with WT mice (fiber innervation density, main effect of genotype, $F_{1,4.2}=9.24, p=0.036$; puncta size, main effect of genotype, $\left.F_{1, \sim 4.2}=9.24, p=0.043\right) . n=3$ WT, 3 GSKI mice.

Figure 8. GSKI mice are impaired in goal-directed learning. WT and GSKI mice underwent a 4 day instrumental conditioning task followed by outcome devaluation to assay goal-directed learning. A, WT mice (black line) made significantly greater numbers of responses during the RI schedule (x-axis), while GSKI mice (blue line) made significantly fewer responses compared to WT on days 3 and 4 RI schedules [main effect (genotype), $F_{1,13}=5.77, p=0.033$, genotype $x$ schedule: $F_{3,39}=5.92, p=$ 0.002; comparison of estimated marginal means, RI-30 day $3, p=0.0034$; RI-30 day 4, $p=0.0025]$. CRF, continuous reinforcement; RI-15, random interval 15 sec; RI-30, random interval $30 \mathrm{sec}$. B, WT (grey lines, left) but not GSKI mice (blue lines, right) demonstrated goal-directed outcome association by their sensitivity to outcome devaluation (valued condition, $\mathrm{V}=$ chow; versus devalued condition, $\mathrm{DV}=$ strawberry 
milk) [main effect (genotype), $F_{1,11}=8.65, p=0.013$; genotype $x$ condition: $F_{1,11}=$ 4.641, $p=0.054$; comparison of estimated marginal means, WT chow vs SM $p=$ 0.0085, GSKI chow vs SM $p=0.98]$. $n=7$ WT, 6 GSKI mice for data shown in A, B. In subsequent tests of motivation for the reward, neither breakpoints in a PR4 task (C) nor reward collection latencies (D) differed between genotypes (breakpoints: Welch's t-test, $\mathrm{t}_{5.68}=0.14, \mathrm{p}=0.89$; latencies: Welch's t-test, $\left.\mathrm{t}_{5.83}=0.38, \mathrm{p}=0.72\right) . \mathrm{n}=4 \mathrm{WT}$, 4 GSKI mice for data shown in C, D.

Figure 9. Pairwise visual discrimination and reversal learning, a measure of cognitive flexibility, is not significantly different in GSKI mice in comparison with WT mice. A, GSKI mice reached performance criteria during both the visual discrimination phase (Vis Dis) and subsequent reversal learning phase (Reversal) over a similar number of sessions in comparison with WT mice (main effect of genotype and interactions of genotype with session and testing phase, Fs $<1$, ps $>0.35$ ). B, there were no genotype differences observed in session length during visual discrimination (Vis Dis) (main effect of genotype $F_{1, \sim 23.1}=$ $.0048, p=0.95$; genotype $x$ testing phase, $\left.\left.F_{1, \sim 213.1}=.1 .57, p=0.21\right)\right]$. C, Mice made more correction trials overall in the reversal phase compared to the initial discrimination phase, $F_{1, \sim 202.1}=73.3, p<0.0001$, but there was no main effect of genotype on correction responses, $F_{1, \sim 53.7}=.035, p=0.85$, or genotype $x$ phase interaction, $F_{1, \sim 202.1}=2.77, p=0.098$, suggesting stimulus-reward learning is intact in GSKI mice. $\mathrm{n}=5 \mathrm{WT}, 7 \mathrm{GSKI}$ mice. 


\section{Figure 1}
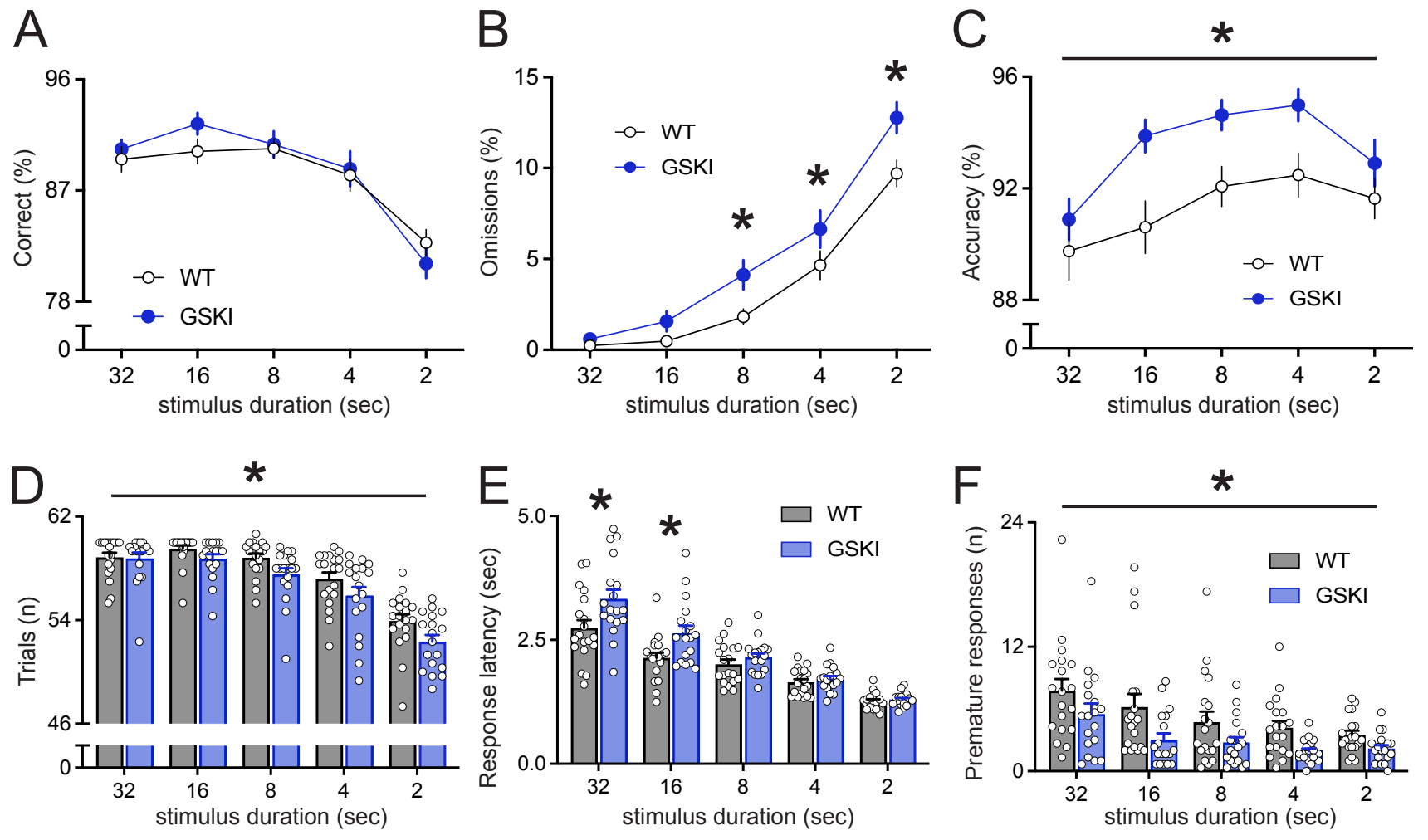

Figure 1. Training performance of WT and GSKI mice in the 5-CSRT task. A, WT (black lines) and GSKI (blue lines) showed similar percentages of correct responses across all stimulus durations [main effect (genotype): $F 1,35=0.195, p=0.66$ ]. $B$, GSKI mice made significantly more omissions [main effect (genotype): $\mathrm{F} 1,35=11.26, \mathrm{p}=0.0019$; genotype $\mathrm{x}$ stimulus duration, $\mathrm{F} 4,510=2.72, \mathrm{p}=0.029$; comparisons of estimated marginal means, $8 \mathrm{sec}, \mathrm{p}=0.0035$; $4 \mathrm{sec}, p=0.012 ; 2 \mathrm{sec}, p=0.0001]$. C, GSKI mice showed significantly greater accuracy in comparison with WT mice [main effect (genotype): $F 1,35=8.62, p=0.005$, but no genotype $x$ stimulus duration interaction, $F 4,510=1.58, p=0.18]$. D, GSKI mice (blue bars) completed fewer trials compared to WT mice (grey bars) [main effect (genotype): F1,35 $=5.78, p=$ 0.022 , but no genotype $x$ stimulus duration interaction, $F 4,510=1.92, p=0.11$. $E$, GSKI mice showed significantly longer correct response latencies in comparison with WT mice [main effect (genotype): $\mathrm{F} 1,35=5.94, \mathrm{p}=0.02$; genotype $\mathrm{x}$ stimulus duration interaction, $\mathrm{F} 4,510=$ $7.43, p<.0001$; comparisons of estimated marginal means, $32 \mathrm{sec}, p<0.0001 ; 16 \mathrm{sec}, p=$ $0.0005]$. F, GSKI mice made significantly fewer premature responses in comparison with WT mice [main effect (genotype): $F 1,35=8.38, p=0.0065$, but no genotype $x$ stimulus duration interaction, $\mathrm{F} 4,510=0.67, \mathrm{p}=0.61] . \mathrm{n}=19 \mathrm{WT}, 18 \mathrm{GSKI}$ mice. 


\section{Figure 2}
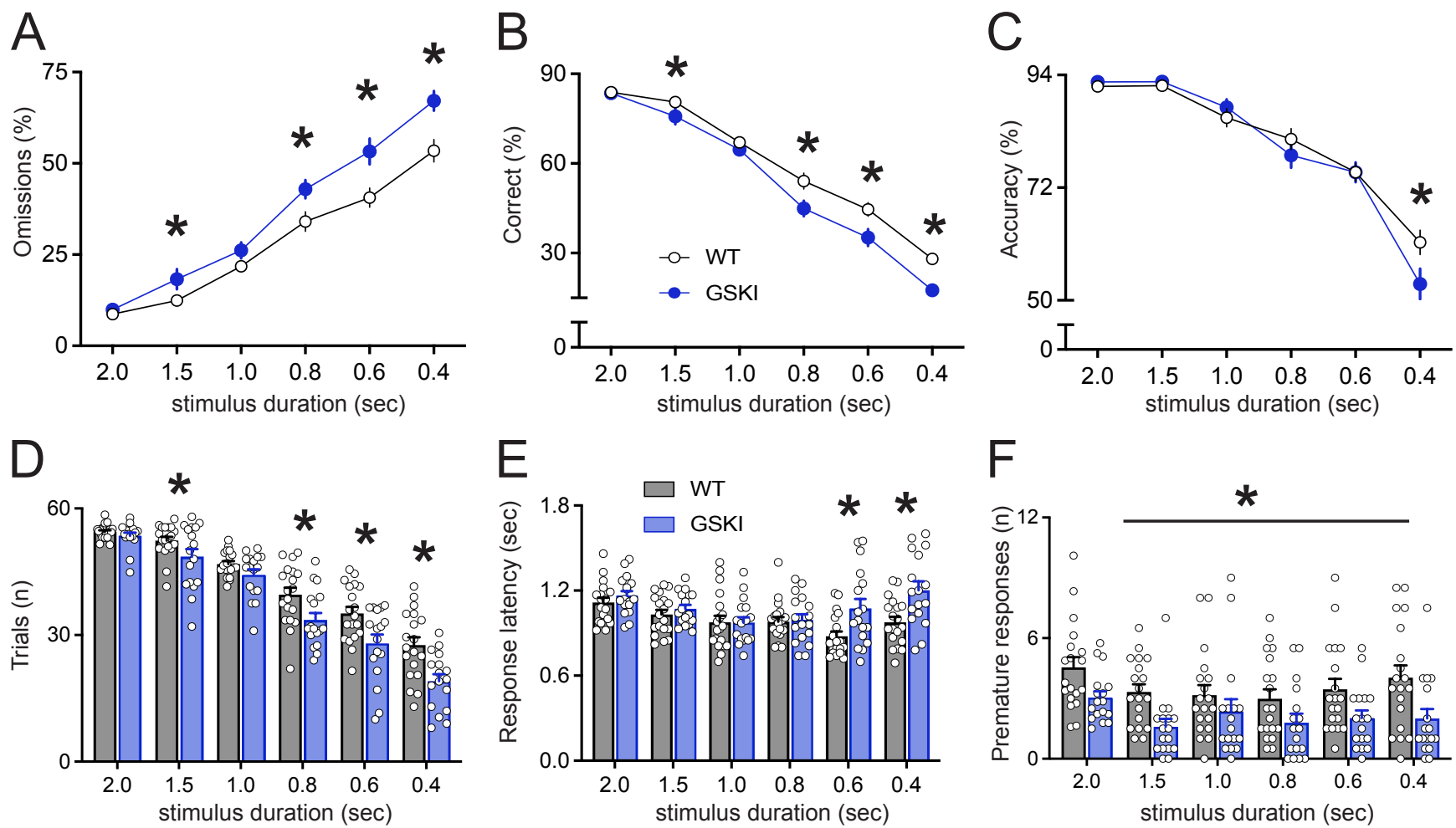

Figure 2. Performance of WT and GSKI mice on probe trial 1 in the 5-CSRT task. A, GSKI mice (blue lines) showed a greater percentage of omissions in comparison with WT mice (black lines) [main effect (genotype): $\mathrm{F} 1, \sim 42.9=36.3, \mathrm{p}<0.0001$, stimulus duration $\times$ genotype: $\mathrm{F} 5, \sim 676.7=9.72, \mathrm{p}<0.0001$; comparisons of estimated marginal means, $1.5 \mathrm{sec}, \mathrm{p}=$ $.014 ; 0.8 \mathrm{sec}, \mathrm{p}=0.0002 ; 0.6$ and $0.4 \mathrm{sec}, \mathrm{p}<0.0001]$. B, GSKI mice showed a decrease in percentage of correct responses at the shortest stimulus duration [main effect (genotype): $F 1, \sim 40.1=17.4, p=0.0002$, stimulus duration $x$ genotype: $F 5, \sim 676.4=8.06, p<0.0001$; comparisons of estimated marginal means, $1.5 \mathrm{sec}, p=0.047 ; 0.8 \mathrm{sec}, p=0.0002 ; 0.6 \mathrm{sec}$, $p=0.0001,0.4 \mathrm{sec}, p<0.0001]$. C, WT and GSKI showed similar accuracy across all stimulus durations except the shortest $(0.4 \mathrm{sec}$ ) [main effect (genotype): F1, 42.5 =1.33, $\mathrm{p}=$ 0.25 , stimulus duration $x$ genotype: $F 5, \sim 677=5.59, p<0.0001$; comparisons of estimated marginal means, $0.4 \mathrm{sec}, p<0.0001]$. D, GSKI mice (blue bars) completed fewer trials in comparison with WT mice (grey bars) [main effect (genotype): F1, 41.7 = 31.7, p $<0.0001$, stimulus duration $x$ genotype: $\mathrm{F} 5, \sim 676.7=8.94, p<0.0001$; comparisons of estimated marginal means, $1.5 \mathrm{sec}, p=0.01 ; 0.8 \mathrm{sec}, p=0.0001 ; 0.6$ and $0.4 \mathrm{sec}, p<0.0001]$. E, GSKI mice showed significantly longer correct response latencies at shorter stimulus durations [main effect (genotype): $\mathrm{F} 1, \sim 39.3=5.37, \mathrm{p}=0.026$, stimulus duration $\mathrm{x}$ genotype: F5, 676.9 $=4.70, p=0.0003$; comparisons of estimated marginal means, $0.6 \mathrm{sec}, p=0.0008 ; 0.4 \mathrm{sec}$, $p=0.0001]$. F, GSKI mice made significantly fewer premature responses during the ITI [main effect (genotype): $\mathrm{F} 1, \sim 44.5=8.99, \mathrm{p}=0.0044]$. $\mathrm{n}=19 \mathrm{WT}, 17 \mathrm{GSKI}$ mice. 


\section{Figure 3}
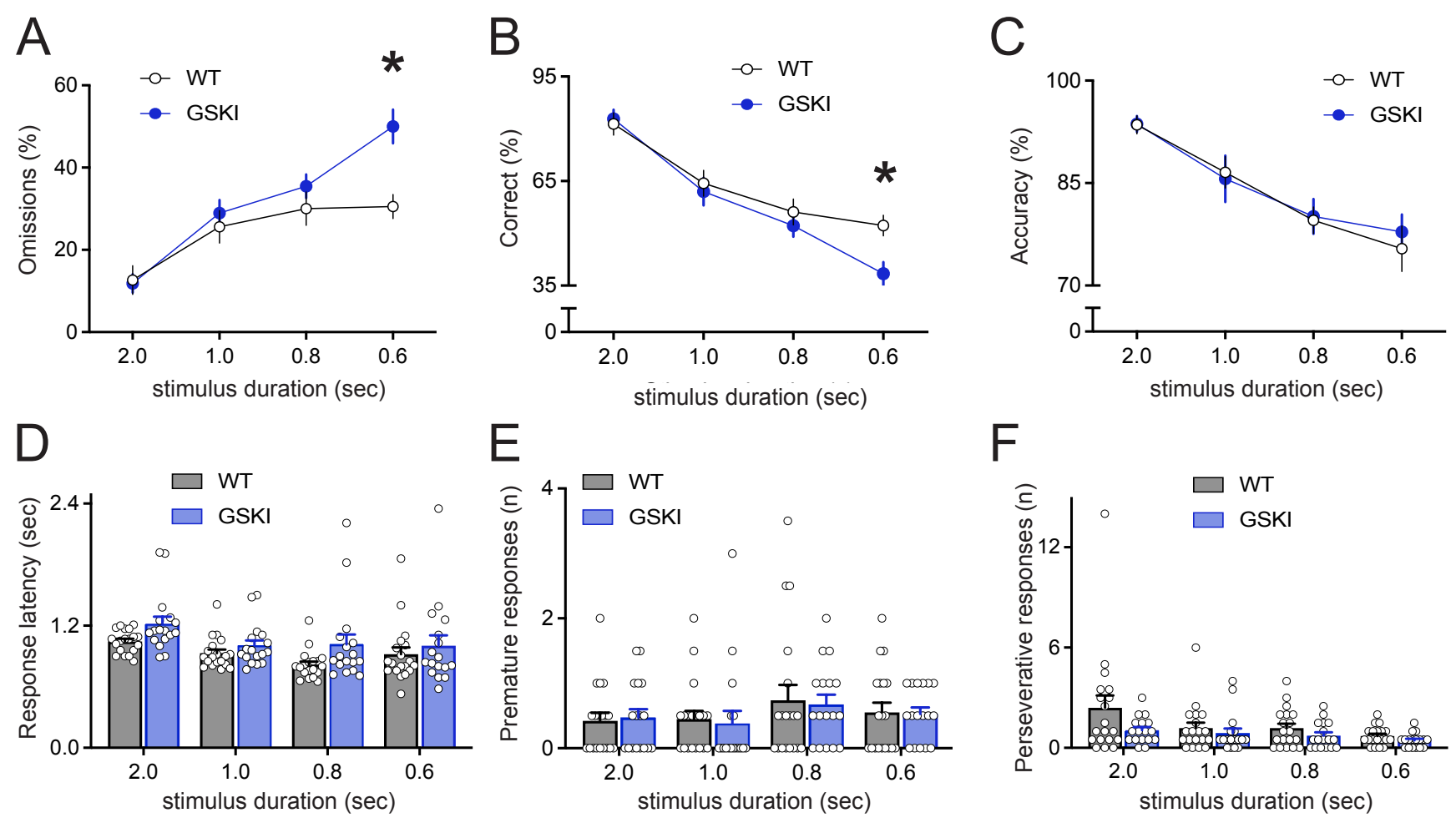

Figure 3. Performance of WT and GSKI mice on probe trial 2 in the 5-CSRT task. A, GSKI mice (blue line) displayed significantly more omissions at the shortest stimulus duration in comparison with WT mice (black line) [stimulus duration $x$ genotype: $F 3,246=8.47, p$ $<0.0001$; comparisons of estimated marginal means, $0.6 \mathrm{sec}, \mathrm{p}=0.0001]$. B, GSKI mice showed a decreased percentage of correct responses at the shortest stimulus duration [stimulus duration $x$ genotype: $F 3,246=4.50, p=0.0043$; comparisons of estimated marginal means, $0.6 \mathrm{sec}, \mathrm{p}=0.0001$ ]. C, GSKI and WT mice showed similar accuracy [main effect (genotype): $F 1,34=0.052, p=0.82$; stimulus duration $x$ genotype: $F 3,246=0.25, p=0.86]$. $D$, GSKI mice (blue bars) showed similar correct response latencies in comparison with WT mice (grey bars) [main effect (genotype): $\mathrm{F} 1,34=3.38, p=0.075$; stimulus duration $\times$ genotype: $\mathrm{F} 3,246=0.73, p=0.54]$. $E$, There were no significant differences between genotypes in premature responses [main effect (genotype): $F 1,34=0.024, p=0.88$; stimulus duration $x$ genotype: $F 3,246=0.066, p=0.98$ ] nor in $(F)$ perseverative responses [main effect (genotype): $F 1,34=3.88, p=0.057$; stimulus duration $x$ genotype: $F 3,246=1.20, p=0.31] . n=$ 13 WT, 11 GSKI mice. 


\section{Figure 4}

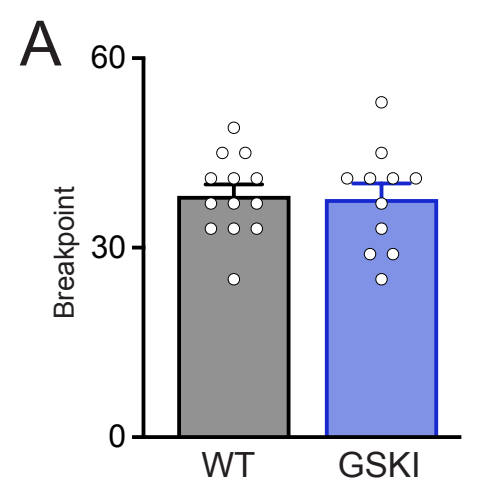

C
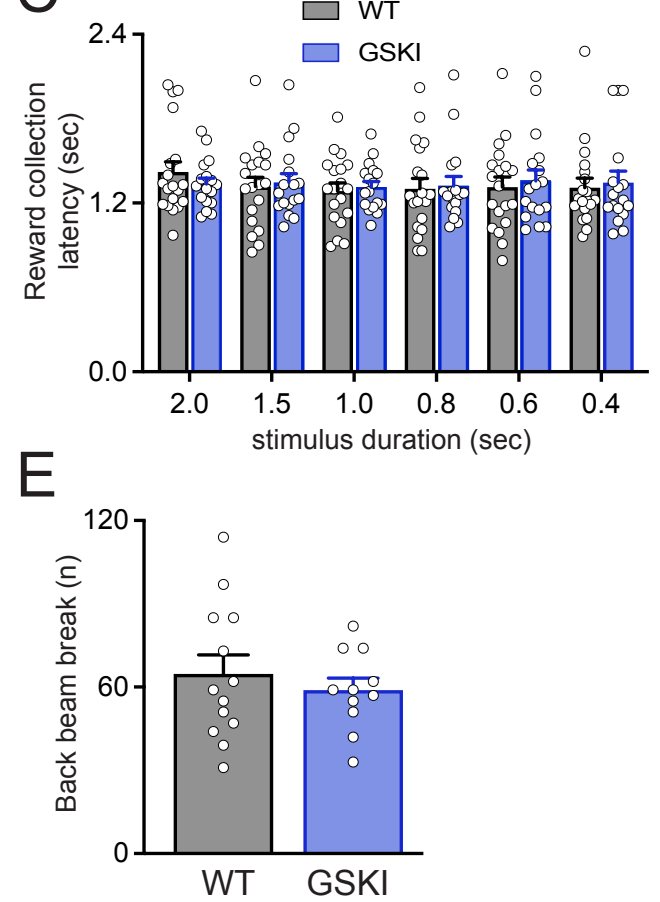

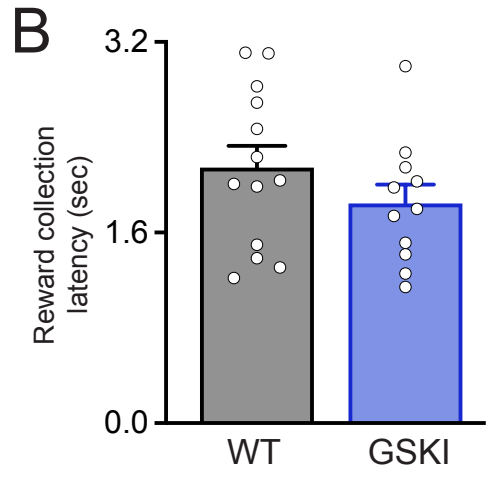

$\mathrm{D}$
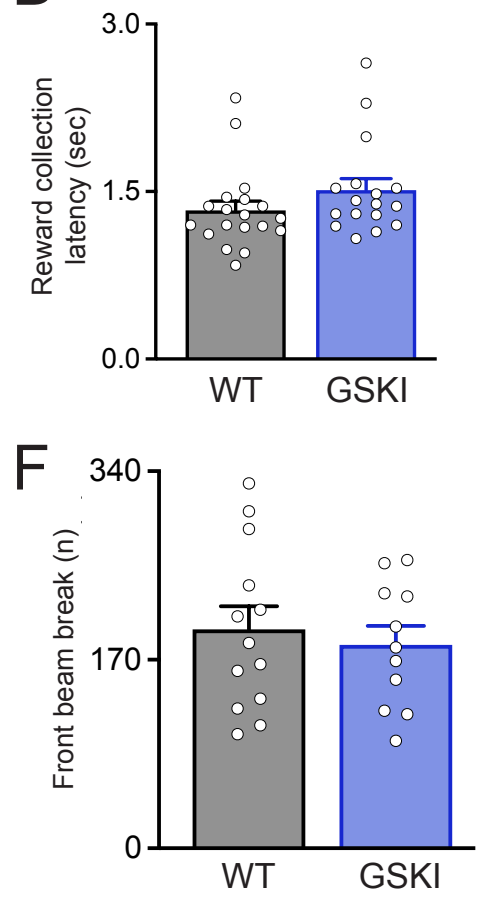

Figure 4. No differences between genotypes in motivation for reward-related behaviors or locomotor activity. In a progressive ratio (PR4) task to test for motivation, the breakpoints $(A)$ and reward collection latencies $(B)$ were similar between genotypes (breakpoints: Welch's t-test, t18.87 $=0.166, p=0.87$; latencies: Welch's t-test, t21.93 $=1.23, p=0.23$ ). Similarly, there were no differences between genotypes in reward collection latencies in probe $1[\mathrm{C}$, main effect (genotype): $\mathrm{F} 1, \sim 42.3=0.046$, $p=0.83]$ or probe $2(D$, Welch's t-test, t31.7 $=1.37, P=0.18)$ in the 5-CSRT task $(n=13$ WT, 11 GSKI mice) in the 5-CSRT. There were no differences between genotypes in numbers of back beam breaks ( $E$, Welch's t-test, $\mathrm{t} 19.73=0.73, \mathrm{p}=0.48$ ) or front beam breaks $(F$, Welch's t-test, t21.76 $=0.53, p=0.6)$ during the progressive ratio task, suggesting no differences in locomotion. For the progressive ratio task, $\mathrm{n}$ = $13 \mathrm{WT}, 11 \mathrm{GSKI}$ mice. 


\section{Figure 5}
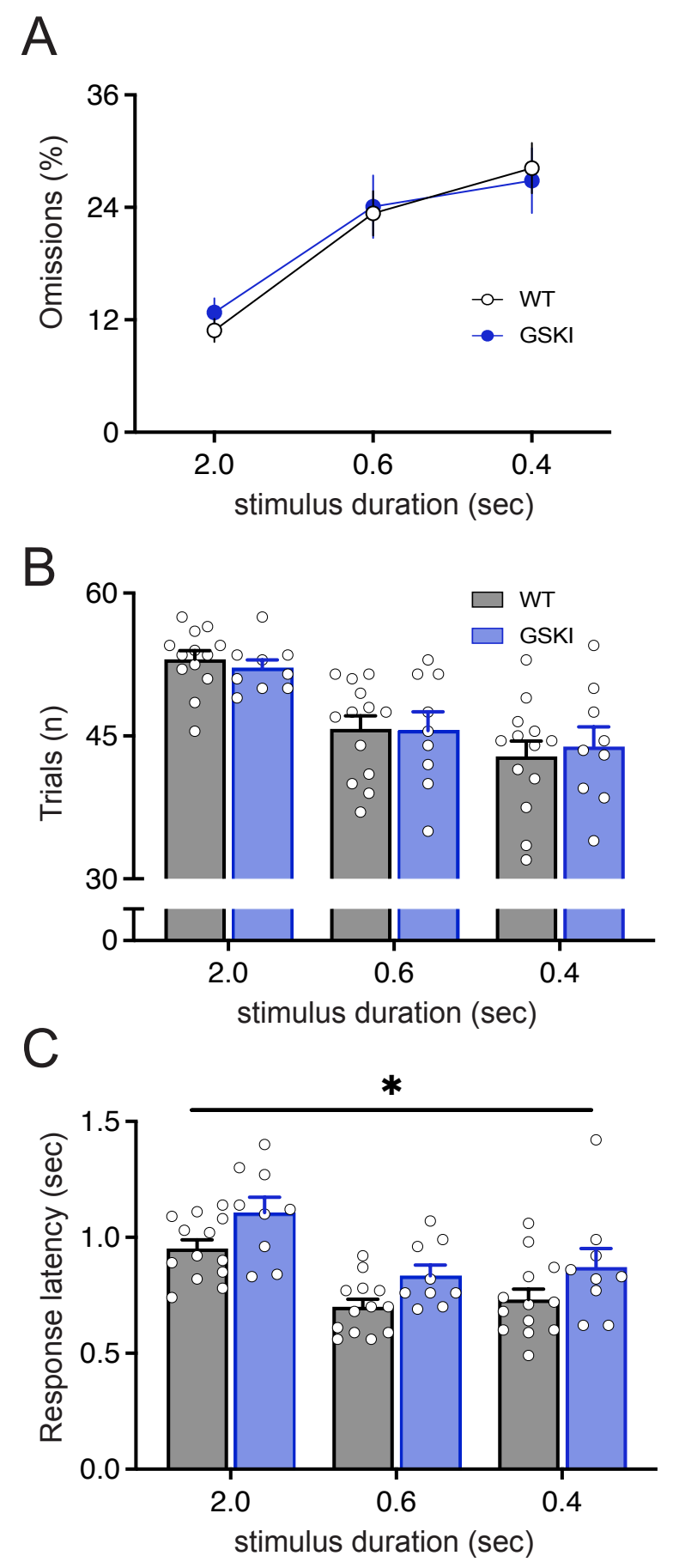

Figure 5. Reducing attentional demand in a 1-choice version of the serial reaction time task normalizes performance deficits in GSKI mice observed at the shortest stimulus durations. There were no differences between genotypes in percent omissions [main effect (genotype): $F 1,20=0.0304$, $p=0.86$; stimulus duration $x$ genotype: $\mathrm{F} 2,106=0.40, p=0.67$ ] or in number of completed trials [main effect (genotype): F1,20 $=0.0002, p=0.99$ stimulus duration $x$ genotype: $F 2,106=0.35, p=$ $0.70]$ in the 1-choice task. There remained a main effect of genotype on correct response latency (C) similar to the 5-CSRT, with GSKI mice showing significantly longer response latencies [main effect (genotype): $\mathrm{F} 1,20=5.24, \mathrm{p}=0.033] . \mathrm{n}=13 \mathrm{WT}, 9 \mathrm{GSKI}$ mice. 


\section{Figure 6}
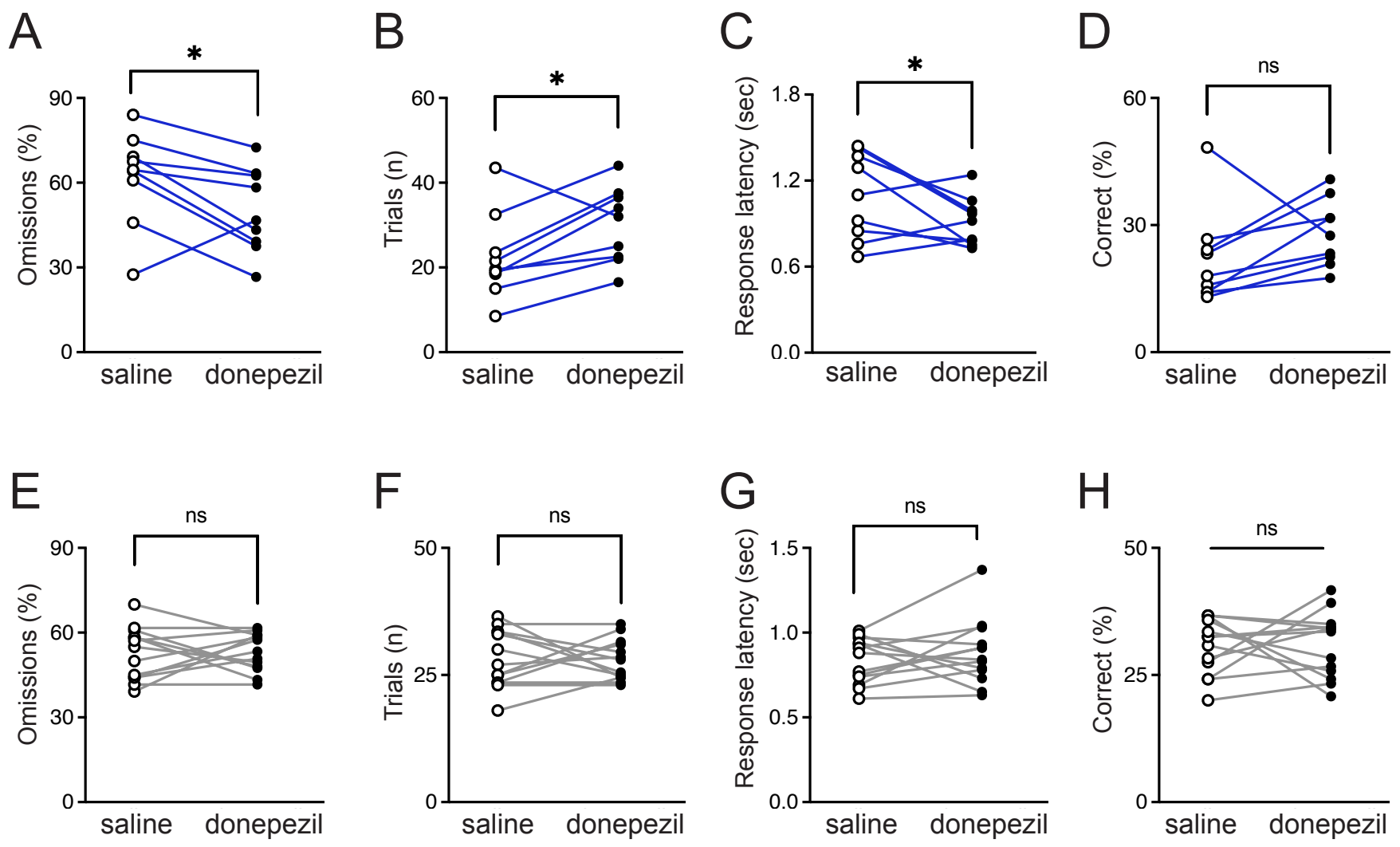

Figure 6. Systemic administration of donepezil, an acetylcholinesterase inhibitor, ameliorated performance deficits of the GSKI mice in the 5 -CSRT task. Donepezil $(0.3 \mathrm{mg} / \mathrm{kg}$, IP) or saline was administered to GSKI mice (blue lines, A-D) or WT mice (grey lines, E-H), using a within-subject design, $1 \mathrm{hr}$ before testing in the 5-CSRT task with a $0.4 \mathrm{sec}$ stimulus duration. Compared to saline treatment (open circles), GSKI mice treated with donepezil (black circles) made significantly fewer omissions (A, genotype $x$ treatment: $F 1,64=5.72, p=0.020$; comparisons of estimated marginal means, GSKI saline vs donepezil, $p=0.003$; $E$, WT saline vs donepezil, $p=0.97$ ) and showed an increased number of completed trials (B, genotype $x$ treatment: $F 1,64=6.47, p=0.013$; comparisons of estimated marginal means, GSKI saline vs donepezil, $p=0.0016$; F, WT saline vs donepezil, $p=0.98$ ). Treating GSKI mice with donepezil lowered correct response latencies compared to saline treatment $(C$, genotype $x$ treatment: $F 1,64=5.91, p=0.018$; comparisons of estimated marginal means, GSKI saline vs donepezil, $p=0.016 ; F$, WT saline vs donepezil, $p=0.41)$. There was no effect of donepezil on percent correct responses $(D$, genotype $x$ treatment: $F 1,64=2.15, p=$ 0.15). In contrast to GSKI mice, donepezil treatment in WT mice had no significant effects (ns) on omissions $(E)$, number of completed trials $(F)$, response latencies $(G)$ or percent correct responses $(\mathrm{H})$ in comparison with saline treatment. $n=13 \mathrm{WT}, 9 \mathrm{GSKI}$ mice. 


\section{Figure 7}
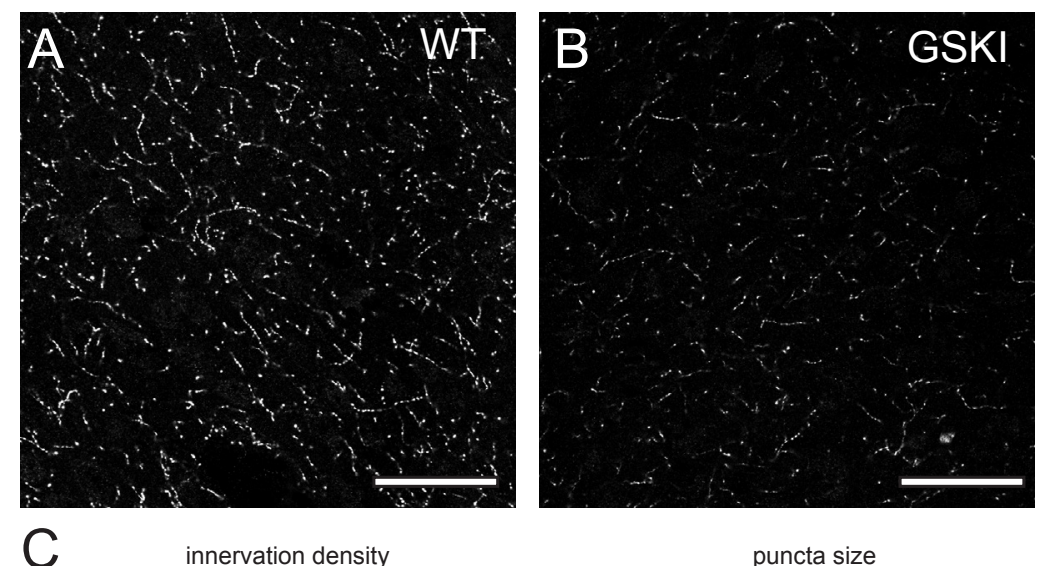

C

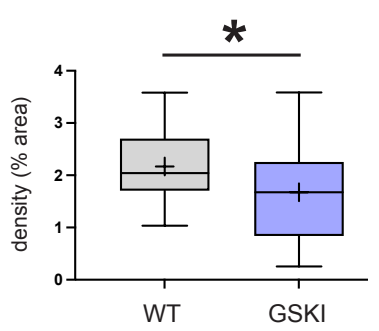

puncta size
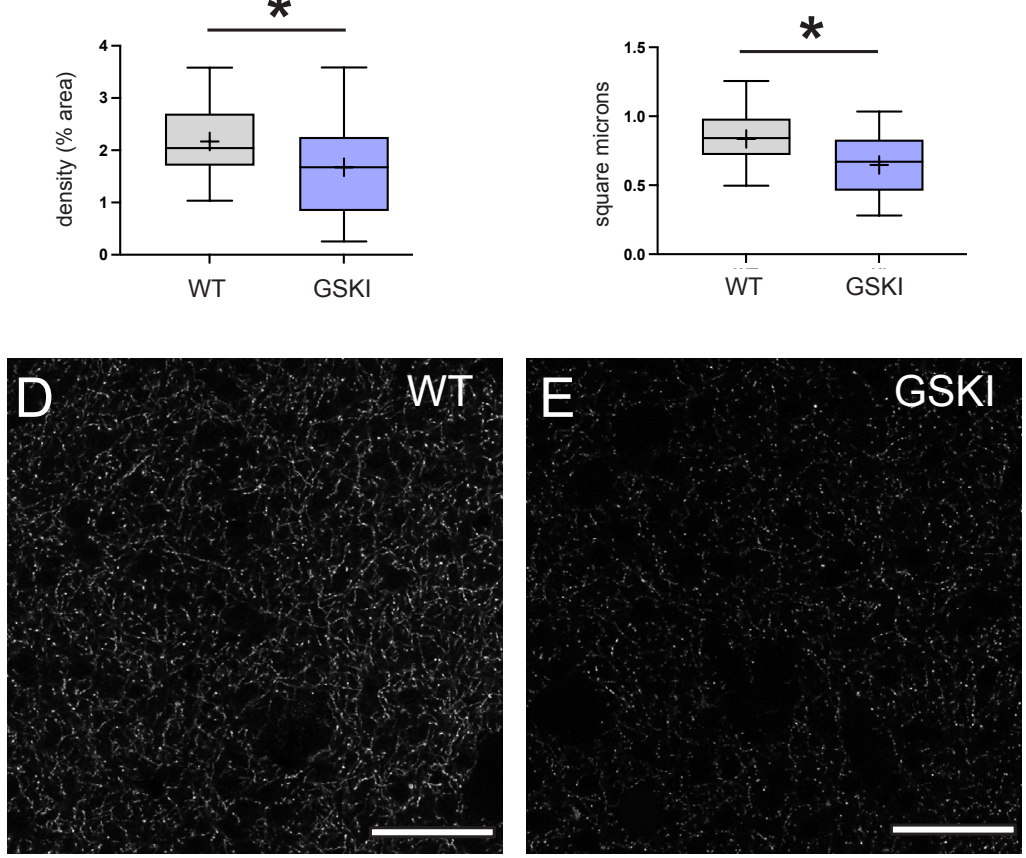

$\mathrm{F}$

innervation density

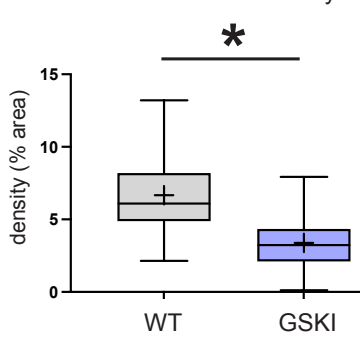

puncta size

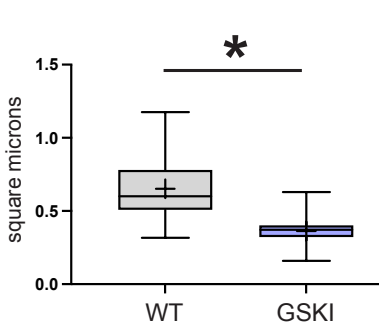

Figure 7. GSKI mice show altered pattern of cholinergic fiber innervation in mPFC and dorsomedial striatum in comparison with WT mice. Representative confocal microscope images of VAChT immunofluorescent labeling of cholinergic fibers in L5 of mPFC areas PL/IL (A, B) or dorsomedial striatum $(\mathrm{D}, \mathrm{E})$ in WT (left images of pair) or GSKI mice (right images of pair), as indicated. bars $=50 \mu \mathrm{m}$. $\mathrm{C}$, $\mathrm{F}$, Box-plots show that in PL/IL $(\mathrm{C})$ and in dorsomedial striatum $(\mathrm{F})$, fiber innervation density is significantly sparser and VAChT-labeled puncta size is significantly smaller in GSKI mice in comparison with WT mice (fiber innervation density, main effect of genotype, F1, 4.2 = 9.24, $p=0.036$; puncta size, main effect of genotype, F1, 4.2 =9.24, $p=0.043)$. $n=3$ WT, 3 GSKI mice. 


\section{Figure 8}
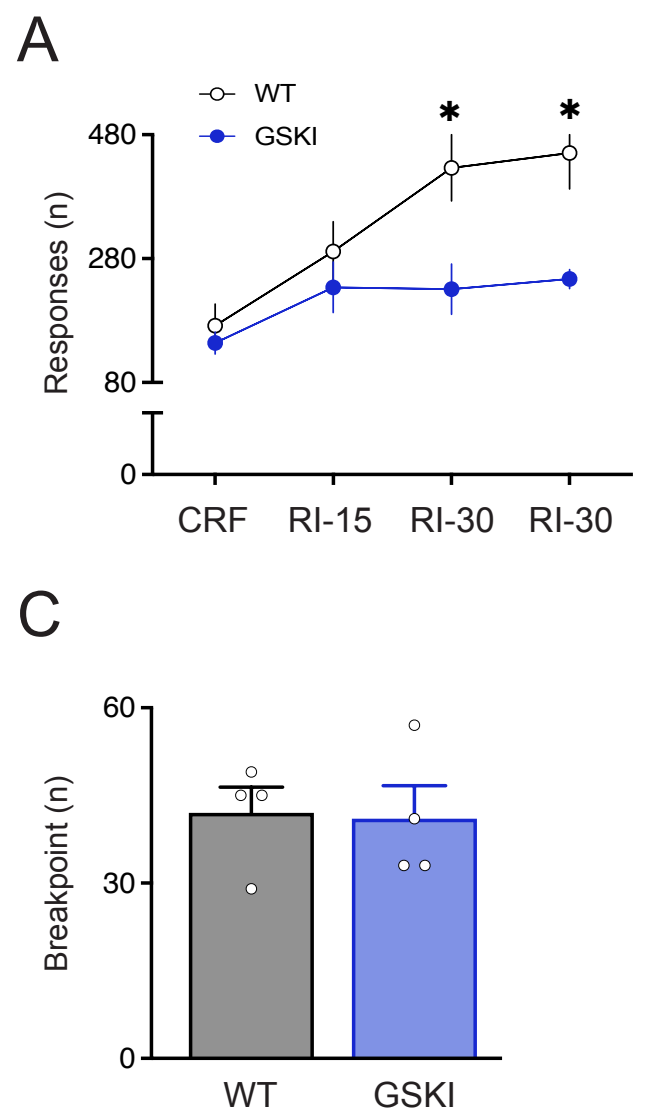
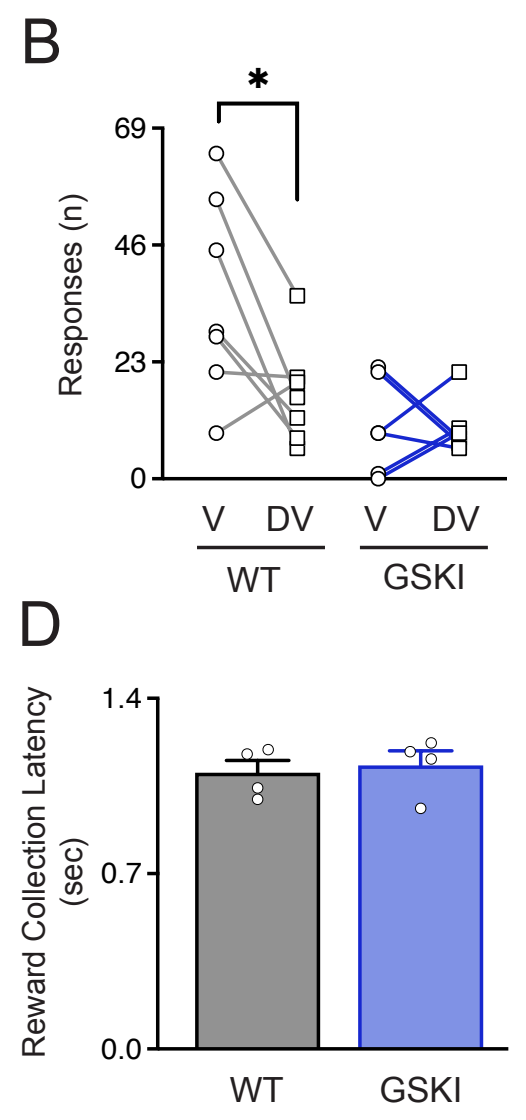

Figure 8. GSKI mice are impaired in goal-directed learning. WT and GSKI mice underwent a 4 day instrumental conditioning task followed by outcome devaluation to assay goal-directed learning. A, WT mice (black line) made significantly greater numbers of responses during the RI schedule (x-axis), while GSKI mice (blue line) made significant ly fewer responses compared to WT on days 3 and 4 RI schedules [main effect (genotype), F1,13 $=5.77, p=0.033$, genotype $x$ schedule: F3,39 $=5.92, p=$ 0.002; comparison of estimated marginal means, RI-30 day $3, p=0.0034$; RI-30 day 4, $p=0.0025$. CRF, continuous reinforcement; RI-15, random interval $15 \mathrm{sec}$; RI-30, random interval $30 \mathrm{sec}$. B, WT (grey lines, left) but not GSKI mice (blue lines, right) demonstrated goal-directed outcome association by their sensitivity to outcome devaluation (valued condition, $V=$ chow; versus devalued condition, $\mathrm{DV}=$ strawberry milk) [main effect (genotype), $\mathrm{F} 1,11=8.65, \mathrm{p}=0.013$; genotype $\mathrm{x}$ condition: $\mathrm{F} 1,11=$ 4.641, $p=0.054$; comparison of estimated marginal means, WT chow vs SM $p=0.0085$, GSKI chow vs $S M p=0.98$ ]. $n=7$ WT, 6 GSKI mice for data shown in A, B. In subsequent tests of motivation for the reward, neither breakpoints in a PR4 task (C) nor reward collection latencies (D) differed between genotypes (breakpoints: Welch's t-test, t5.68 $=0.14, p=0.89$; latencies: Welch's t-test, t5.83 $=0.38, p$ $=0.72) . n=4$ WT, 4 GSKI mice for data shown in C, D. 


\section{Figure 9}
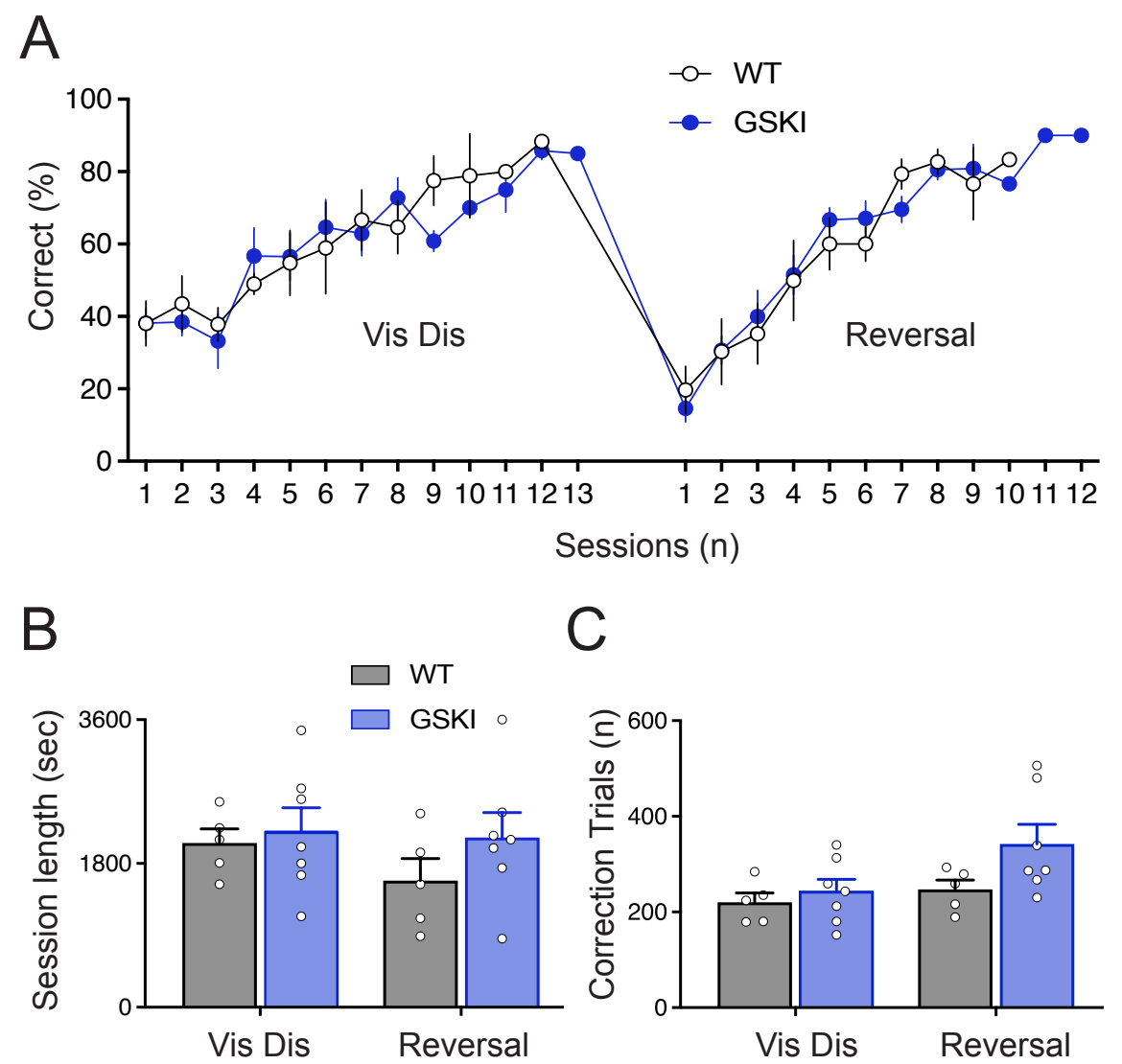

C

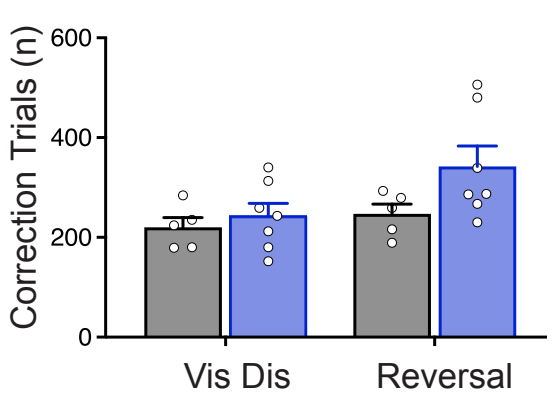

Figure 9. Pairwise visual discrimination and reversal learning, a measure of cognitive flexibility, is not significantly different in GSKI mice in comparison with WT mice. A, GSKI mice reached performance criteria during both the visual discrimination phase (Vis Dis) and subsequent reversal learning phase (Reversal) over a similar number of sessions in comparison with WT mice (main effect of genotype and interactions of genotype with session and testing phase, Fs $<1$, ps $>0.35$ ). B, there were no genotype differences observed in session length during visual discrimination (Vis Dis) (main effect of genotype $\mathrm{F} 1, \sim 23.1=.0048, \mathrm{p}=0.95$; genotype $\mathrm{x}$ testing phase, $\mathrm{F} 1, \sim 213.1=.1 .57, \mathrm{p}=0.21)]$. $\mathrm{C}$, Mice made more correction trials overall in the reversal phase compared to the initial discrimination phase, $\mathrm{F} 1, \sim 202.1=73.3, p<0.0001$, but there was no main effect of genotype on correction responses, $F 1, \sim 53.7=.035, p=0.85$, or genotype $x$ phase interaction, $F 1, \sim 202.1=2.77, p=0.098$, suggesting stimulus-reward learning is intact in GSKI mice. $n=5$ WT, 7 GSKI mice. 